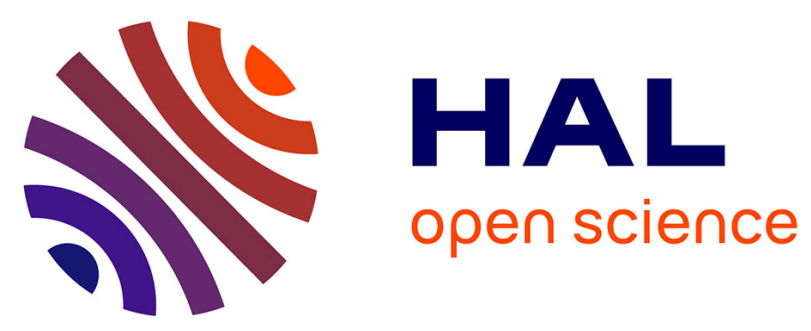

\title{
Mutual Successive Interference Cancellation Strategies in NOMA for Enhancing the Spectral Efficiency of CoMP Systems
}

Antoine Kilzi, Joumana Farah, Charbel Abdel Nour, Catherine Douillard

\section{To cite this version:}

Antoine Kilzi, Joumana Farah, Charbel Abdel Nour, Catherine Douillard. Mutual Successive Interference Cancellation Strategies in NOMA for Enhancing the Spectral Efficiency of CoMP Systems. IEEE Transactions on Communications, 2020, 68 (2), pp.1213-1226. 10.1109/TCOMM.2019.2945781 . hal02311284

\section{HAL Id: hal-02311284 \\ https://imt-atlantique.hal.science/hal-02311284}

Submitted on 10 Oct 2019

HAL is a multi-disciplinary open access archive for the deposit and dissemination of scientific research documents, whether they are published or not. The documents may come from teaching and research institutions in France or abroad, or from public or private research centers.
L'archive ouverte pluridisciplinaire HAL, est destinée au dépôt et à la diffusion de documents scientifiques de niveau recherche, publiés ou non, émanant des établissements d'enseignement et de recherche français ou étrangers, des laboratoires publics ou privés. 


\title{
Mutual Successive Interference Cancellation Strategies in NOMA for Enhancing the Spectral Efficiency of CoMP Systems
}

\author{
Antoine Kilzi, Joumana Farah, Charbel Abdel Nour, Catherine Douillard
}

\begin{abstract}
The densification of mobile networks should enable the fifth generation (5G) mobile networks to cope with the ever increasing demand for higher rate traffic, reduced latency, and improved reliability. The large scale deployment of small cells and distributed antenna systems in heterogeneous environments will require more elaborate interference mitigating techniques to increase spectral efficiency and to help unlock the expected performance leaps from the new network topologies. Coordinated multi-point (CoMP) is the most advanced framework for interference management enabling the cooperation between base stations to mitigate inter-cell interference and boost cell-edge user performance. In this paper, we study the combination of CoMP with mutual SIC, an interference cancellation technique based on power-domain non-orthogonal multiple access (NOMA) that enables multiplexed users to simultaneously cancel their corresponding interfering signals. A highly efficient intercell interference cancellation scheme is then devised, that can encompass several deployment configurations and coordination techniques. The obtained results prove the superiority of this approach compared to conventional NOMA-CoMP systems.
\end{abstract}

Index Terms-Distributed antenna systems, non orthogonal multiple access, coordinated multipoint, successive interference cancellation, mutual SIC.

\section{INTRODUCTION}

$\mathbf{O}$ VER the last 5 years, an eighteenfold increase in mobile data traffic was observed according to [1]. The tendency is expected to last as the data traffic forecasts revolve around a compound annual growth rate (CAGR) of $46 \%$ for the 5 years to come, resulting in a sevenfold increase. Moreover, the diversification of services with the emergence of internet of things (IoT), machine to machine (M2M), vehicle to vehicle $(\mathrm{V} 2 \mathrm{~V})$ communications and other technologies require a greater flexibility from the operators to meet all sorts of demands. To cope with the presented challenge, new multiple access techniques, such as the non-orthogonal multiple access (NOMA), and new network architectures are being investigated for the fifth generation $(5 \mathrm{G})$ of cellular systems, like distributed antenna systems (DAS) [2]-[4], cloud radio access networks (CRAN) [5], [6], and small cells [7], [8], leading to heterogeneous networks (HetNets) [9], [10] which are needed to match the diverse demands. Although frequency reuse patterns and, hence

J. Farah is with the Department of Electricity and Electronics, Faculty of Engineering, Lebanese University, Roumieh, Lebanon (joumanafarah@ul.edu.lb).

A. Kilzi, C. Abdel Nour and C. Douillard are with Institut Mines-Telecom, CNRS UMR 6285 Lab-STICC, 29238 Brest, France, (email: antoine.kilzi@imtatlantique.fr; charbel.abdelnour@imt-atlantique.fr; catherine.douillard@imtatlantique.fr).

This work has been funded with support from IMT Atlantique and the Lebanese University. the network planning, differ from an architecture/technology to another, the common driving idea remains the densification of the network deployment to drastically enhance signal quality by reducing the mean path-loss, the shadowing effect and localizing the inter/intra-cell interference.

Recently, NOMA has gained much attention as a promising multiple access technique, in both academia and industry, due to its potential in increasing the spectral efficiency and/or the number of accommodated user equipments, without requiring additional time and frequency resources [11]-[15]. Although several types of NOMA have been investigated over the last few years, an important portion of the work revolved around power domain NOMA. Its fundamental idea is to enable the simultaneous access of multiple users to the same time/frequency resources, by allocating different power levels to users, based on the user channel gains. At the receiver side, each user extracts its own signal by successively demodulating, decoding then re-encoding before subtracting the successively detected interfering signals. According to [16], the successive interference cancellation (SIC) ordering that maximizes the sum rate for downlink in single base station (BS) cells is done according to the ascending order of the user channel gains. That is, each NOMA user decodes and subtracts the signals of all other users with weaker channel gains before retrieving its intended signal. Hereinafter, we refer to power domain NOMA simply by NOMA.

A direct result of conventional SIC ordering in conventional NOMA systems is that cell-edge users suffer from the intracell interference of other multiplexed users in the cell, and are more vulnerable to inter-cell interference (ICI) due to their location. To mitigate the ICI of both orthogonal multiple access (OMA) and NOMA systems, the third generation partnership project (3GPP) proposed in release 9 [17] and then adopted in release 11 [18] coordinated multipoint (CoMP) as the evolution of enhanced ICI coordination (eICIC) to improve the performance of interference-prone users. With CoMP, cooperating cells can share the channel state information (CSI) of users when scheduling is performed. This sharing makes joint scheduling possible. Different CoMP techniques have been proposed in [19], [20]. In coordinated scheduling (CS-CoMP) and coordinated beamforming (CB-CoMP), CSI is shared while user data is not shared among the cooperating transmission points (TP); hence user data is only available at one TP. On the other hand, more elaborate CoMP techniques such as dynamic point selection (DPS-CoMP) and joint transmission (JT-CoMP) require enough backhaul capacity so that user information is 
available at every TP of the CoMP BSs. In JT-CoMP, signals are transmitted to the user from multiple TPs, therefore tighter synchronization between JT cells is required.

Several studies have proposed the combination of NOMA with CoMP techniques. In [21], the authors study a CoMPNOMA system for downlink transmission and propose a suboptimal scheduling strategy that scales linearly with the number of users. In [22], the applicability of different NOMACOMP scenarios is studied. The authors also argue that signals of users receiving CoMP transmissions must be decoded prior to those of non-CoMP users receiving single transmission. In [23], CoMP scenarios are studied in a HetNet system consisting of a macro BS, and multiple small BSs. The users requiring JT-CoMP transmission are first determined according to the received signal strength (RSS). Users with weak RSS - cell-edge users - are granted JT-CoMP transmission. The sub-optimal user-clustering for NOMA users developed in [16] is adopted, then a low complexity distributed power allocation for rate maximization is performed independently on every BS. In [24], a two-cell system made of one cell-edge user and two cell-center users (one in each cell) is studied. Alamouti code is utilized with joint transmission to serve the cell-edge user with JT-CoMP in order to improve the performance of this user.

In all previous studies on NOMA-CoMP, only cell-edge users are considered as potential CoMP users. Also, cell-edge users are not considered able to decode and remove the signals of inner cell users. Finally, user-antenna association for nonCoMP users is based on the sole criterion of maximal RSS or channel gain. In a previous work, we studied the use of NOMA in a DAS network, and exploited the combination of NOMA with DAS to tackle the downlink power minimization problem under fixed user rates [25], and also under RRH-specific transmit power constraints [26]. We showed that, under some channel and transmit power conditions, when multiplexed signals are sent from different RRHs, paired users can both cancel the interference of each other, hence the name mutual SIC. Such a configuration corresponds in fact to an intra-site CoMP (using DPS), behaving as inter-site CoMP [20]. This new concept of mutual SIC relying on CoMP systems makes the combination of NOMA and CoMP much more interesting than their combination using the single SIC approach. Indeed, a complete interference cancellation (intra-cell and inter-cell) among users from the same NOMA cluster (whether cell-edge or cell-center users) becomes possible. Therefore, we study in this paper the combination of NOMA and multicell-CoMP, establishing the conditions enabling a successful mutual SIC procedure at the level of all users, and assessing the performance by means of the system throughput metric.

In the current study, we consider the case of mutual SIC in a two-cell system and enlarge the scope of NOMA application to JT-CoMP. The key contributions of this paper can be summarized as follows:

- We propose to improve cell-edge user rate and overall system throughput by introducing JT not only for celledge but also for cell-centered users. In practice, JT is not necessarily applied to all users on all sub-bands, but may be restricted to users signals transmitted on sub-bands including at least one cell-edge user.

- We study the conditions for allowing interference cancellation in NOMA for both DPS and JT scenarios, and show that SIC of the signals of inner users is possible at the level of the cell-edge user.

- We rigorously define the conditions allowing the feasibility of mutual SIC for any user and apply it to a three-user NOMA cluster.

- We show that JT is more favorable for enabling interference cancellation than DPS without being a necessary condition for achieving mutual SIC.

- We challenge the common practice of max-RSS-based user-antenna association for achieving the highest system capacity, and we favor the associations allowing the much more profitable mutual SIC procedure.

The paper is organized as follows: The system model is given in Section II. In Section III, the fundamental conditions for the establishment of mutual SIC in CoMP, for the general case of two randomly chosen users, are derived. Next, Sections IV and $\mathrm{V}$ describe the followed methodology to take advantage of the mutual SIC procedure whenever possible, for 2-user and 3 -user clusters. The results of throughput maximization by a proper power allocation allowing mutual SIC are presented and interpreted in Section VI. The major conclusions are drawn in Section VII.

\section{SySTEM MODEL}

We consider a two-cell downlink system where each cell has multiple RRHs deployed in a DAS setup such that the RRHs are connected to the base band units (BBU) through high capacity optical fibers (see Fig. 1). In this work, singleantenna RRHs are considered; therefore, the terms "RRH" and "antenna" will be used interchangeably. Users transmit their CSI to RRHs, and the BBU collects all the CSI from RRHs and shares them with other BBUs. Perfect CSI is assumed in this study (the influence of imperfect channel estimation is out of the scope of this work). Inter-BBU message exchange can be done through a direct X2 link between the BBUs of the two cells, in case of a fully meshed decentralized CoMP architecture, or both BBUs may be connected to a third party central unit in a star-like network, for a centralized CoMP architecture. In any case, whether the central unit coordinates the BBUs (which in turn control the RRHs), or whether BBUs exchange information in a decentralized manner, we assume that the information data relative to any user is made available at the level of both BBUs of each cell. Therefore, a joint processing (JP) CoMP scenario is considered with either a DPS scheme, where users are served by one antenna at a time, or a JT scheme in which a user may benefit from the transmission of the same signal over multiple antennas at the same time. To focus on the cell-edge user scenario, we restrict the choice of serving antennas to the two located near the common frontier of the cells, one on each side. Let $\mathcal{K}$ be the set of users, with a maximum number of three users considered in the system, user 1 being a non-cell-edge user present in cell 1, user 2 a non-cell-edge user located in cell 2, and user 
3 the cell-edge user. The serving RRH for user 1 in cell 1 is referred to as $r_{1}$ and that of user 2 in cell 2 is referred to as $r_{2}$. The system framework is presented in the schematic of Fig.1. Without loss of generality, three different geometric regions were defined, in which each user is randomly positioned.

The problem structure of this study is radically different from that in [25], since the purpose of this work is to showcase the important advantages of combining mutual SIC with CoMP, rather than devising new resource allocation schemes. Indeed, in a practical implementation with a significant number of users in each cell, appropriate pairing or clustering methods must be incorporated in the resource allocation technique, so as to assign NOMA clusters of 2 or 3 users to sub-bands [4], [16], [25], [27]. This study is therefore focused on one of these particular clusters. More specifically, the main objective of this paper is the study of the conditions enabling the combination of mutual SIC and CoMP, with the focus directed to upper layer aspects of SIC (physical aspects of mutual SIC are out of the scope of the paper). The resulting enhancements of the service quality of users in general, and cell-edge users in particular, are compared against classic NOMA scenarios [11], [13], [15], or previous CoMP scenarios [24]. To do so, the performance of different CoMP systems is analyzed from the system capacity perspective. We aim to maximize the achievable total throughput of any given configuration, under the following sets of constraints:

- SIC constraints: the set of conditions that make the mutual SIC technique possible from the information theory perspective, i.e. the conditions on achievable rates at the respective users levels.

- Power multiplexing constraints (PMC): the set of conditions that make the mutual SIC technique feasible from a practical implementation perspective, i.e. the conditions on the received signals powers at the respective users levels. Let $s_{i}$ be the signal of user $i, i \in\{1,2,3\}$. According to the SIC principle, if signals $s_{1}, s_{2}$ and $s_{3}$ are to be decoded in that order at the level of one of the users, then the signal power of $s_{1}$ at the level of that user must be greater than that of $s_{2}$ and $s_{3}$ combined, and the power level of $s_{2}$ must be greater than that of $s_{3}$. This guarantees SIC



Fig. 1: Illustration of the two-cell DAS setup with the functional RRHs $r_{1}$ and $r_{2}$, and the three colored user regions (UE $=$ user equipment) stability since every signal is ensured to be the dominant signal during its decoding [16], [28].

- Power limit constraints: the maximum total amount of transmit power available at the level of the RRHs.

Let $h_{k, r}$ be the channel attenuation experienced by a signal between antenna $r$ and user $k$. In this study, a frequency-nonselective channel is assumed, so that $h_{k, r}$ is independent from the sub-band frequency, therefore it only accounts for pathloss and shadowing. However, the study can be directly adapted to the case of frequency-selective channels, by incorporating the channel short-term variations within $h_{k, r}$. In this case, additional sub-band allocation techniques, such as the ones developed in [25] or [4], need to be considered, which is beyond the scope of the current paper. Let $P_{k, r}$ be the power of the message $s_{k}$ transmitted from antenna $r$ to user $k$. The signal transmitted by $r$ reaches $k$ after experiencing the channel with attenuation factor $h_{k, r}$; the received signal power is therefore $P_{k, r} h_{k, r}$. In the case of JT, both antennas $r_{1}$ and $r_{2}$ are used for the transmission of the message to $k$ with their transmit powers being respectively $P_{k, r_{1}}$ and $P_{k, r_{2}}$. Hence, the received signal power is the sum of the received powers from both antennas, i.e. $P_{k, r_{1}} h_{k, r_{1}}$ from $r_{1}$, and $P_{k, r_{2}} h_{k, r_{2}}$ from $r_{2}$, totaling $P_{k, r_{1}} h_{k, r_{1}}+P_{k, r_{2}} h_{k, r_{2}}$. The system throughput is the sum of the rates achieved by all users in the system, its expression depending on whether DPS or JT is adopted and on the intra-cell and inter-cell interfering terms. When there are no interference (which is the case with a full mutual SIC between the three users), the rate expression for a user $k$ is given by the Shannon capacity theorem:

$$
R_{k}=\left\{\begin{array}{l}
B \log _{2}\left(1+\frac{P_{k, r} h_{k, r}}{\sigma^{2}}\right) \text { for DPS } \\
B \log _{2}\left(1+\frac{P_{k, r_{1}} h_{k, r_{1}}+P_{k, r_{2}} h_{k, r_{2}}}{\sigma^{2}}\right) \text { for JT, }
\end{array}\right.
$$

$\sigma^{2}$ is the noise power and $B$ is the signal bandwidth normalized to one. The problem formulation of sum-rate maximization over the transmit power variables $P_{k, r}$ takes the following generic form:

$$
\max _{P_{k, r}} \sum_{i \in \mathcal{K}} R_{i}
$$

such that:

$$
\left\{\begin{array}{l}
\text { Mutual SIC constraints are verified, } \\
\text { PMCs are verified, } \\
\text { Power limit constraints are verified. }
\end{array}\right.
$$

In the following section, we derive the fundamental mutual SIC constraints for a general system of $M$ users and two transmitting RRHs in a CoMP scenario. Then, attention is directed towards the application of the mutual SIC technique in a two-user and a three-user system in Sections IV and $\mathrm{V}$ respectively. The expressions of $(3 \mathrm{~b}),(3 \mathrm{c})$ and $(3 \mathrm{~d})$ will therefore be developed in each case.

\section{Mutual SIC CONDitions FOR COMP ScEnARIos}

In this section, we study the conditions, in terms of channel coefficients and transmit powers, that must be met to enable 
the mutual SIC procedure at the level of all users for any NOMA cluster size. To this end, a general framework for identifying the interfering user sets depending on the decoding order is introduced. The developed methodology is provided for JT transmission scenario which encompasses simpler DPS transmission schemes. In other words, the conditions concerning DPS-based mutual SIC schemes can be easily adapted from those shown in this section by canceling the transmitted power from one of the antennas to the user.

Let $\mathcal{M}$ be the NOMA cluster with dimension $M$, i.e. $\mathcal{M}$ is the set of users multiplexed over the same frequency resource. Given two users $p$ and $n$ randomly selected in that cluster, we seek to determine the conditions under which a successful mutual SIC can occur between the two users while in the presence of interfering signals from the remaining users in $\mathcal{M}$. In [25], the mutual SIC conditions were developed for the special case of two users per cluster and a single-cell system. The rate conditions that must be verified to guarantee mutual SIC can be translated into signal-to-interference-plus-noise ratio (SINR) conditions: For $n$ to successfully decode (and cancel) the signal of $p$ denoted by $s_{p}$, the SINR of $s_{p}$ at the level of $n$, denoted by $S I N R_{n}^{s_{p}}$ must be greater than the SINR of $s_{p}$ at the level of $p$ itself $\left(S I N R_{p}^{s_{p}}\right)$. Therefore, the conditions of mutual SIC at the level of both users are:

$$
\begin{cases}S I N R_{p}^{s_{n}}>S I N R_{n}^{s_{n}} & \text { SIC of } s_{n} \text { at user } p, \\ S I N R_{n}^{s_{p}}>S I N R_{p}^{s_{p}} & \text { SIC of } s_{p} \text { at user } n,\end{cases}
$$

Determining the SINRs requires the knowledge of the interfering signals at the level of every user, at the time of decoding signals $s_{n}$ and $s_{p}$. For example, if $p$ has been managed to decode the signal of a third user $m$ in the cluster while $n$ has not been, the SINR of $p$ will not suffer from the interference caused by $s_{m}$, while decoding either $s_{n}$ or $s_{p}$. The same cannot be said for user $n$ in that case. This remark highlights the importance of the decoding order at every user. Indeed, the SINR terms vary according to this decoding order, which is instructed by the BBU to the RRH and then to the user via signaling. Therefore, mutual SIC conditions depend on each possible decoding order. Let $\mathcal{I}_{p}$ and $\mathcal{I}_{n}$ be the sets of interfering users on users $p$ and $n$ respectively. $\mathcal{I}_{p}$ and $\mathcal{I}_{n}$ can be each partitioned into two sets, a set of common interfering users between $n$ and $p$ named $\mathcal{C}_{p n}$, and a set of interfering users specific to $n$ and $p, \mathcal{U}_{n}$ and $\mathcal{U}_{p}$ respectively. These sets have the following properties:

$$
\begin{gathered}
\mathcal{I}_{p}=\mathcal{C}_{p n} \cup \mathcal{U}_{p}, \quad \mathcal{C}_{p n} \cap \mathcal{U}_{p}=\emptyset, \\
\mathcal{I}_{n}=\mathcal{C}_{p n} \cup \mathcal{U}_{n}, \quad \mathcal{C}_{p n} \cap \mathcal{U}_{n}=\emptyset, \\
\mathcal{U}_{p} \cap \mathcal{U}_{n}=\emptyset
\end{gathered}
$$

Depending on whether $s_{n}$ or $s_{p}$ is considered for decoding in (4) or (5) respectively, the interfering signals are interchanged between $s_{n}$ and $s_{p} . s_{n}$ is the useful signal in (4), and becomes the interfering signal in (5), whereas $s_{p}$ is the interfering signal in (4) and the useful signal in (5). Therefore, the interfering user sets depend upon the signal that is being decoded and their notation must be defined accordingly. Also, since $s_{n}$ is a common interfering signal to users $p$ and $n$ in (5), $n$ belongs to $\mathcal{C}_{p n}$ when decoding $s_{p}$ and the same applies to $p$ when decoding $s_{n}$ in (4). The complete notations are as follows:

$$
\text { for (4) }\left\{\begin{array} { l } 
{ \mathcal { I } _ { p } ^ { s _ { n } } = \mathcal { C } _ { p n } ^ { s _ { n } } \cup \mathcal { U } _ { p } , } \\
{ \mathcal { I } _ { n } ^ { s _ { n } } = \mathcal { C } _ { p n } ^ { s _ { n } } \cup \mathcal { U } _ { n } , }
\end{array} \quad \text { for (5) } \left\{\begin{array}{l}
\mathcal{I}_{p}^{s_{p}}=\mathcal{C}_{p n}^{s_{p}} \cup \mathcal{U}_{p}, \\
\mathcal{I}_{n}^{s_{p}}=\mathcal{C}_{p n}^{s_{p}} \cup \mathcal{U}_{n},
\end{array}\right.\right.
$$

The superscripts in $\mathcal{I}$ and $\mathcal{C}$ refer to the signal that is being decoded. Note that $\mathcal{U}_{p}$ and $\mathcal{U}_{n}$ remain unchanged. Therefore, the expression of $S I N R_{p}^{s_{n}}$ can be written as:

$$
\begin{gathered}
S I N R_{p}^{s_{n}}=\frac{P_{n, 1} h_{p, 1}+P_{n, 2} h_{p, 2}}{\sum_{i \in \mathcal{I}_{p}^{s_{n}}}\left(P_{i, 1} h_{p, 1}+P_{i, 2} h_{p, 2}\right)+\sigma^{2}} \\
=\frac{P_{n, 1} h_{p, 1}+P_{n, 2} h_{p, 2}}{\sum_{i \in \mathcal{C}_{p n}^{s_{n}}}\left(P_{i, 1} h_{p, 1}+P_{i, 2} h_{p, 2}\right)+\sum_{i \in \mathcal{U}_{p}}\left(P_{i, 1} h_{p, 1}+P_{i, 2} h_{p, 2}\right)+\sigma^{2}} .
\end{gathered}
$$

With these notations in hand, the mutual SIC conditions that derive from (4) and (5) can now be developed by comparing $S I N R_{p}^{s_{n}}$ with $S I N R_{n}^{s_{n}}$ in (4), and $S I N R_{n}^{s_{p}}$ with $S I N R_{p}^{s_{p}}$ in (5). The details of these calculations can be found in Appendix A. The fundamental results are the following: To cancel the interference of signal $s_{n}$ at the level of user $p$, the channel coefficients and transmit powers must meet the following constraint:

$$
\begin{gathered}
h_{n, 1} h_{p, 1} P_{n, 1}\left[\sum_{i \in \mathcal{U}_{n}} P_{i, 1}-\sum_{i \in \mathcal{U}_{p}} P_{i, 1}\right] \\
+h_{n, 2} h_{p, 2} P_{n, 2}\left[\sum_{i \in \mathcal{U}_{n}} P_{i, 2}-\sum_{i \in \mathcal{U}_{p}} P_{i, 2}\right] \\
+\left(h_{p, 1} h_{n, 2}-h_{p, 2} h_{n, 1}\right)\left[P_{n, 1} \sum_{i \in \mathcal{C}_{p n}^{s_{n}}} P_{i, 2}-P_{n, 2} \sum_{i \in \mathcal{C}_{p n}^{s_{n}}} P_{i, 1}\right] \\
+h_{p, 1} h_{n, 2}\left[P_{n, 1} \sum_{i \in \mathcal{U}_{n}} P_{i, 2}-P_{n, 2} \sum_{i \in \mathcal{U}_{p}} P_{i, 1}\right] \\
+h_{p, 2} h_{n, 1}\left[P_{n, 2} \sum_{i \in \mathcal{U}_{n}} P_{i, 1}-P_{n, 1} \sum_{i \in \mathcal{U}_{p}} P_{i, 2}\right]>0 .
\end{gathered}
$$

To determine the condition for the decoding of $s_{p}$ at the level of user $n, n$ and $p$ are simply swapped in (6).

Having defined the general conditions of a mutual SIC between two users of a NOMA cluster of size $M$, we consider the special cases $M=2$ and $M=3$ in the following sections. We explore the specific properties of every case allowing different mutual SIC scenarios, establish the corresponding set of PMCs, discuss their significance and implications, before describing the followed methodology to efficiently assess the performance of each scenario.

\section{Mutual SIC in a Two-User System}

To determine the mutual SIC conditions in a two-user system, we first have to identify the interfering user sets for each user. Without loss of generality, we consider in this section that only users 1 and 2 from Fig. 1 are present in the system. However, the same reasoning can be developed for any couple of users, whether it includes a cell-center user and a cell-edge user 
or two cell-center users, leading to the same conditions with different indexes. Since users 1 and 2 constitute the whole NOMA cluster, the interfering sets specific to each user, $\mathcal{U}_{1}$ and $\mathcal{U}_{2}$, are empty and the interfering sets $\mathcal{I}_{1}$ and $\mathcal{I}_{2}$ are identical. Thus, by letting $p=1$ and $n=2$, we get $\mathcal{C}_{12}^{s_{2}}=\{1\}$, and the condition (6) under which user 1 is capable of decoding the signal $s_{2}$ of user 2 becomes:

$$
\left(h_{1,1} h_{2,2}-h_{1,2} h_{2,1}\right)\left[P_{2,1} P_{1,2}-P_{2,2} P_{1,1}\right]>0 .
$$

Also, by letting $p=2$ and $n=1$, we get $\mathcal{C}_{21}^{s_{1}}=\{2\}$, and the condition under which user 2 is capable of decoding the signal $s_{1}$ of user 1 is:

$$
\left(h_{2,1} h_{1,2}-h_{1,1} h_{2,2}\right)\left[P_{1,1} P_{2,2}-P_{1,2} P_{2,1}\right]>0 .
$$

These two SIC conditions are equivalent and form a unique constraint. Therefore, if one user satisfies the constraint of interference cancellation, the other one does as well, and if one cannot perform SIC, the other user cannot either. This result is radically different from that of classic SIC in a centralized antenna system (CAS) [11], [13], [15], or a DAS with the paired signals powered by a common RRH (this case was studied in [25]), where only one user out of the two performs interference cancellation.

Next, we investigate mutual SIC in DPS and JT scenarios. We highlight the PMCs that differentiate each case as well as the corresponding formulation of the power limit constraints, before defining the new user-RRH association and power allocation strategy in each case.

\section{A. Two-user system with DPS}

1) DPS-MutSIC: The use of multiple antennas to power the signals of multiplexed users is what rendered feasible the mutual SIC procedure that we introduced in [25]. The only transmission scenario considered in [25] is in fact an intra-site CoMP with dynamic point selection only. As stated earlier, the calculation developed in Appendix A considers the general case of JT-served users. To obtain the underlying DPS constraints, the signal must be transmitted from one antenna only. This translates into canceling out either $P_{m, 1}$ or $P_{m, 2}$ for any user $m(m=1$ or 2$)$. By setting $P_{2,1}$ and $P_{1,2}$ to 0 , user 1 is assigned to $r_{1}$ and user 2 to $r_{2}$, and the mutual SIC condition (7) becomes solely dependent upon the channel coefficients of the users, i.e. :

$$
P_{2,2} P_{1,1}\left(h_{1,2} h_{2,1}-h_{1,1} h_{2,2}\right)>0 \Rightarrow h_{1,2} h_{2,1}>h_{1,1} h_{2,2} .
$$

In other terms, the ability of the system to perform mutual SIC when user 1 is powered by $r_{1}$ and user 2 by $r_{2}$ is uniquely determined by the channel characteristics of the system, since the power factors are necessarily positive. However, if $h_{1,1} h_{2,2}>h_{1,2} h_{2,1}$, mutual SIC can still be achieved in the system by switching the serving antennas of the users. Indeed, if $P_{1,1}=P_{2,2}=0$ in (7), then user 1 is served by $r_{2}$ and user 2 by $r_{1}$, satisfying the new corresponding mutual SIC constraints as follows:

$$
P_{2,1} P_{1,2}\left(h_{1,1} h_{2,2}-h_{1,2} h_{2,1}\right)>0 \Rightarrow h_{1,1} h_{2,2}>h_{1,2} h_{2,1} .
$$

As a conclusion, in a two-user system using DPS, the channel characteristics are the only factor that determines the antenna association of each user: if $h_{1,2} h_{2,1}>h_{1,1} h_{2,2}$, user 1 is served by $r_{1}$ and user 2 by $r_{2}$; if not, the antenna association is simply reversed. Note that in either case, the users are not necessarily assigned their best antenna, from the channel gain perspective. For example, considering the case where $h_{1,2} h_{2,1}>h_{1,1} h_{2,2}$, it is impossible to have $h_{1,1}>h_{1,2}$ and $h_{2,2}>h_{2,1}$ at the same time, meaning that at least one user will not be served by its best antenna. Either only one user is assigned to its most preferable antenna, or neither user is served by its best antenna. Therefore, the mutual SIC procedure goes against the usual practice of associating the user to its closest/best antenna. While this might seem counter-intuitive at first, it should be understood that the rate gain provided by interference cancellation greatly overcomes the channel gain "deficit", as shown in Section VI.

Moving on to the power multiplexing constraints, the power allocation (PA) must ensure that the power level of the signal to be decoded (at the level of a given user) is higher than the combined power levels of the remaining signals that have not been decoded yet. Table I presents the PMC and power limit constraints for every user according to the channel characteristics. $P_{L_{1}}$ and $P_{L_{2}}$ are the transmit power limits of RRHs $r_{1}$ and $r_{2}$ respectively. The two PMC of the first case

TABLE I: PMC and power limit constraints for two-user DPS clusters

\begin{tabular}{|c|c|c|}
\cline { 2 - 3 } & \multicolumn{2}{|c|}{ Channel gain conditions } \\
\cline { 2 - 3 } & $h_{1,1} h_{2,2}<h_{1,2} h_{2,1}$ & $h_{1,1} h_{2,2}>h_{1,2} h_{2,1}$ \\
\hline User 1 PMC & $P_{2,2} h_{1,2}>P_{1,1} h_{1,1}$ & $P_{2,1} h_{1,1}>P_{1,2} h_{1,2}$ \\
\hline User 2 PMC & $P_{1,1} h_{2,1}>P_{2,2} h_{2,2}$ & $P_{1,2} h_{2,2}>P_{2,1} h_{2,1}$ \\
\hline Power limit & $P_{1,1} \leq P_{L_{1}}$ & $P_{2,1} \leq P_{L_{1}}$ \\
& $P_{2,2} \leq P_{L_{2}}$ & $P_{1,2} \leq P_{L_{2}}$ \\
\hline
\end{tabular}

in Table I can be summed up in the following form:

$$
\frac{h_{2,2}}{h_{2,1}}<\frac{P_{1,1}}{P_{2,2}}<\frac{h_{1,2}}{h_{1,1}} .
$$

Note that a necessary and sufficient condition for the existence of a power allocation scheme that satisfies (10) is to have (8). Indeed if (8) is true, the right member of (10) is greater than the left one. The same holds for the second case in Table I, when (9) is true.

The objective function of the optimization problem (3a) presented in Section II is the sum of the user rates as expressed in (1). The mutual SIC constraints determine the user-antenna association, and this affects the expressions of the PMCs and power limits as shown in Table I. The corresponding strategy will be referred to as DPS-MutSIC. 
2) DPS-NoSIC: To assess the efficiency of DPS-MutSIC, we also consider a benchmark scenario, namely DPS-NoSIC, in which the mutual SIC procedure is excluded at both user sides. Thus, the imposed PMCs for DPS-MutSIC are dropped. In DPS-NoSIC, users may be served by the same antenna as there is no more obligation to satisfy the mutual SIC conditions. Then, for any given channel realization, two additional userantenna associations are identified when both users are served by the same antenna $r_{1}$ or $r_{2}$, which raises to four the number of possible user-antenna associations. The expressions of the users rates now include the interfering term from every other user and are given as follows:

$$
\begin{aligned}
& R_{1}=\log _{2}\left(1+\frac{P_{s_{1}, r\left(s_{1}\right)} h_{1, r\left(s_{1}\right)}}{P_{s_{2}, r\left(s_{2}\right)} h_{1, r\left(s_{2}\right)}+\sigma^{2}}\right), \\
& R_{2}=\log _{2}\left(1+\frac{P_{s_{2}, r\left(s_{2}\right)} h_{2, r\left(s_{2}\right)}}{P_{s_{1}, r\left(s_{1}\right)} h_{2, r\left(s_{1}\right)}+\sigma^{2}}\right),
\end{aligned}
$$

where $r\left(s_{k}\right)$ denotes the antenna powering the signal of user $k$. For every channel realization, the problems corresponding to the four user-antenna associations are solved, and the scheme yielding the highest throughput is retained.

\section{B. Two-user system with JT}

1) JT-MutSIC: Users subject to joint transmission receive their information signals from multiple antennas/cells. In that regard, a user is not associated to a specific cell, and the idea of switching the user-antenna association as done for the DPS case becomes irrelevant. The validity of the mutual SIC constraint is a function of the channel and power variables as opposed to DPS. The BBUs must therefore adapt the PA in order to maintain (7):

$$
\left(h_{1,1} h_{2,2}-h_{1,2} h_{2,1}\right)\left[P_{2,1} P_{1,2}-P_{2,2} P_{1,1}\right]>0 .
$$

By inspecting (7), it can be seen that if $h_{1,1} h_{2,2}>h_{1,2} h_{2,1}$ the PA must ensure that $P_{2,1} P_{1,2}>P_{2,2} P_{1,1}$; otherwise, the power condition must be reversed.

Regarding the PMCs, the power level of $s_{1}$ at the level of user 1 is the sum of the signal powers from $r_{1}$ and $r_{2}$ and it amounts to $P_{1,1} h_{1,1}+P_{1,2} h_{1,2}$. Therefore, the PMC for the decoding of $s_{2}$ at the level of user 1 is given by :

$$
P_{2,1} h_{1,1}+P_{2,2} h_{1,2}>P_{1,1} h_{1,1}+P_{1,2} h_{1,2} .
$$

Similarly, the PMC for the decoding of $s_{1}$ at the level of user 2 is:

$$
P_{1,1} h_{2,1}+P_{1,2} h_{2,2}>P_{2,1} h_{2,1}+P_{2,2} h_{2,2} .
$$

Proposition 1. If the PMCs of a two-user system are valid, the mutual SIC condition is necessarily valid as well.

Proof. Let us rewrite the PMCs (11) and (12) in the following form:

$$
\begin{aligned}
& \left(P_{2,2}-P_{1,2}\right) h_{1,2}>\left(P_{1,1}-P_{2,1}\right) h_{1,1}, \\
& \left(P_{1,1}-P_{2,1}\right) h_{2,1}>\left(P_{2,2}-P_{1,2}\right) h_{2,2} .
\end{aligned}
$$

Then, the terms $P_{1,1}-P_{2,1}$ and $P_{2,2}-P_{1,2}$ have the same sign. If they are both positive, we get the following inequality:

$$
\frac{h_{1,1}}{h_{1,2}} \leq \frac{P_{2,2}-P_{1,2}}{P_{1,1}-P_{2,1}} \leq \frac{h_{2,1}}{h_{2,2}},
$$

this leads to $h_{2,2} h_{1,1}<h_{1,2} h_{2,1}$ (actually, the channel constraint will impose the positive sign of $P_{1,1}-P_{2,1}$ and $P_{2,2}-$ $P_{1,2}$, not the other way around). However, since $P_{1,1}-P_{2,1}$ and $P_{2,2}-P_{1,2}$ are assumed positive, $P_{2,2} P_{1,1}>P_{2,1} P_{1,2}$. The mutual SIC condition (7) is thus verified, since the power term $\left(P_{2,1} P_{1,2}-P_{2,2} P_{1,1}\right)$ and channel term $\left(h_{1,1} h_{2,2}-h_{1,2} h_{2,1}\right)$ have the same sign.

Similarly, assuming the negativity of $P_{1,1}-P_{2,1}$ and $P_{2,2}-P_{1,2}$ implies the opposite channel conditions $\left(h_{1,1} h_{2,2}>h_{1,2} h_{2,1}\right)$ and transmit power relations $\left(P_{2,1}>P_{1,1}\right.$ and $P_{1,2}>P_{2,2} \Longrightarrow$ $P_{1,2} P_{2,1}>P_{1,1} P_{2,2}$ ), which renders (7) a product of two positive terms. This concludes our proof.

Finally, the transmit power constraints at the level of each RRH are as follows:

$$
\begin{aligned}
& P_{1,1}+P_{2,1} \leq P_{L_{1}}, \\
& P_{1,2}+P_{2,2} \leq P_{L_{2}} .
\end{aligned}
$$

On a side note, even though user-antenna association is irrelevant to the JT context, the power allocation is reminiscent of the user-antenna selection in DPS: when $h_{1,2} h_{2,1}>h_{1,1} h_{2,2}$, the dominant signal transmitted by $r_{1}$ is $s_{1}$ (since $P_{1,1}>P_{2,1}$ ) and the dominant signal transmitted by $r_{2}$ is $s_{2}$ (since $P_{2,2}>$ $P_{1,2}$ ), bringing us back to the user-antenna association in DPS when $h_{1,2} h_{2,1}>h_{1,1} h_{2,2}$. The same analysis applies when $h_{1,2} h_{2,1}<h_{1,1} h_{2,2}: s_{2}$ is dominant at the level of $r_{1}$ and $s_{1}$ is dominant at the level of $r_{2}$. This showcases how the DPS is a special case of JT and implies that JT is naturally richer in potential and properties (JT can be seen as the superposition of the 2 DPS configurations). For this reason, in Section V, we consider only JT scenarios for a three-user system, as it inherently encompasses all the DPS cases and many others.

At last, the problem formulation for the JT case can be summed up as: maximize the sum rate $R_{1}+R_{2}$ expressed using (2), under the power limit constraints (13) and (14) and the PMCs (11) and (12).

2) JT-NoSIC: JT-NoSIC is introduced to assess the efficiency of the mutual SIC procedure when applied to JT-users. It serves as benchmark for the performance of JT-MutSIC. The problem structure in JT-NoSIC remains globally unchanged except that the power multiplexing constraints are dropped, and the rate expressions of $R_{1}$ and $R_{2}$ are given by:

$$
\begin{aligned}
& R_{1}=\log _{2}\left(1+\frac{P_{1,1} h_{1,1}+P_{1,2} h_{1,2}}{P_{2,1} h_{1,1}+P_{2,2} h_{1,2}+\sigma^{2}}\right), \\
& R_{2}=\log _{2}\left(1+\frac{P_{2,1} h_{2,1}+P_{2,2} h_{2,2}}{P_{1,1} h_{2,1}+P_{1,2} h_{2,2}+\sigma^{2}}\right) .
\end{aligned}
$$

\section{Mutual SiC in a Three-User System}

In this section, mutual SIC is studied for a three-user NOMA cluster. The conventional technique for serving users is presented first, then a new scheme based on a full JT system is introduced. At last, a middle ground strategy combining the proposed and conventional serving methods is proposed to enable a fair comparison between the methods. 


\section{A. The previous technique: CellEdgeJT-CellCenterSIC}

The conventional way of employing JT was first thought of as a way to improve the signal quality of weak cell-edge users that suffer the most from inter-cell interference. Inner-cell users are generally considered to be more interference-immune given their relative proximity to the serving antenna, and their relative distance from the interfering ones. Along these lines, the study in [24] sought to improve the system spectral efficiency by serving the cell-edge user, user 3 in Fig. (1), by both RRHs $r_{1}$ and $r_{2}$, while user 1 and 2 are served uniquely by their closest antennas, $r_{1}$ and $r_{2}$ respectively. In that setup, the cell-edge user suffers from the interference of both user 1 and user 2; however, it is the only user taking advantage of cell coordination in JT. Users 1 and 2 are able to successfully decode the signal of user 3 but cannot remove each others signals. From a classic single-antenna single-SIC point of view, the cell-edge user is the weak user both in cell 1 with user 1 , and in cell 2 with user 2. We refer to this method as CellEdgeJT-CellCenterSIC. Let us determine the SIC conditions at the level of user 1 and user 2 respectively to remove the signal of user 3 (these conditions were not considered in [24]). Since both users $p=1$ (resp. $p=2$ ) and user $n=3$ suffer from the interference of user $m=2$ (resp. $m=1$ ), then we have $\mathcal{C}_{p n}^{s_{n}}=\{m, p\}=\{2,1\}$ $(\{1,2\}), \mathcal{U}_{p}=\mathcal{U}_{n}=\emptyset$. After replacing each variable by its value in (6), and keeping in mind that $P_{1,2}=P_{2,1}=0$, the SIC conditions for the decoding of $s_{3}$ at the level of users 1 and 2 are respectively:

$$
\begin{aligned}
& \left(h_{1,1} h_{3,2}-h_{1,2} h_{3,1}\right)\left[P_{3,1} P_{2,2}-P_{3,2} P_{1,1}\right]>0, \\
& \left(h_{2,1} h_{3,2}-h_{2,2} h_{3,1}\right)\left[P_{3,1} P_{2,2}-P_{3,2} P_{1,1}\right]>0,
\end{aligned}
$$

these conditions imply that the common power factor and the two channel factors must be of the same sign:

$$
\begin{aligned}
\operatorname{sign}\left(h_{1,1} h_{3,2}-h_{1,2} h_{3,1}\right) & =\operatorname{sign}\left(h_{2,1} h_{3,2}-h_{2,2} h_{3,1}\right) \\
& =\operatorname{sign}\left(P_{3,1} P_{2,2}-P_{3,2} P_{1,1}\right) .
\end{aligned}
$$

The validity of this SIC procedure is mainly based on the channel properties: if the two channel factors are not of the same sign, SIC is not applicable.

The problem formulation of CellEdgeJT-CellCenterSIC resides in the maximization of the sum rate of $R_{1}, R_{2}$ and $R_{3}$ given by:

$$
\begin{gathered}
R_{1}=\log _{2}\left(1+\frac{P_{1,1} h_{1,1}}{P_{2,2} h_{1,2}+\sigma^{2}}\right), \\
R_{2}=\log _{2}\left(1+\frac{P_{2,2} h_{2,2}}{P_{1,1} h_{2,1}+\sigma^{2}}\right), \\
R_{3}=\log _{2}\left(1+\frac{P_{3,1} h_{3,1}+P_{3,2} h_{3,2}}{P_{1,1} h_{3,1}+P_{2,2} h_{3,2}+\sigma^{2}}\right),
\end{gathered}
$$

having (15) as SIC constraints, and the following PMC and power limit constraints :

$$
\begin{gathered}
P_{3,1} h_{1,1}+P_{3,2} h_{1,2}>P_{1,1} h_{1,1}+P_{2,2} h_{1,2}, \\
P_{3,1} h_{2,1}+P_{3,2} h_{2,2}>P_{2,2} h_{2,2}+P_{1,1} h_{2,1}, \\
P_{1,1}+P_{3,1} \leq P_{L_{1}}, \\
P_{2,2}+P_{3,2} \leq P_{L_{2}} .
\end{gathered}
$$

\section{B. Triple mutual SIC in a JT system: FullJT-TripleMutSIC}

In this subsection, we propose the use of JT for the whole NOMA cluster, that is driven by three main reasons:

1) The densification of the network topology implies smaller distances between users and antennas, but also between RRHs of different cells. This proximity of RRHs brings back into question the ICI-immune character of cellcenter users, hence the potential use of JT for these users.

2) Inspired by the results of Section IV-A, the ideas of weak and strong users fade away in the presence of a mutual SIC procedure. Therefore, exploring the mutual SIC capabilities of the system for all three users and not just the cell-edge user is an idea worth investigating.

3) The use of JT maximizes the chances of successful triple mutual SIC, since all possible DPS combinations are only special cases of joint transmission as pointed out in Section IV-B.

We propose a new method to enable a complete mutual SIC procedure at the level of every user, through the use of JT. This means that every user must be able to decode and subtract the signals of the two other users. The mutual SIC conditions, in this case, strongly depend on the decoding order undergone at the level of each user, as previously discussed in Section III. This decoding order is related to the PMCs: user $p$ cannot decode the signal of user $n$ unless the power level of $s_{n}$ is dominant at $p$. At the level of every user, two decoding orders are possible, raising to eight the total number of decoding orders combinations in the system. Table II presents the eight possible decoding orders. The second row labels each combination by an identifying number. The cells of the table indicate, for any user (row), and any selected combination (column), the decoding order followed at the level of the user. For example, in the first combination, user 1 starts by decoding the signal of user 2 before proceeding to that of user 3 .

TABLE II: The eight potential decoding orders of a triple mutual SIC

\begin{tabular}{|c|c|c|c|c|c|c|c|c|}
\cline { 2 - 9 } \multicolumn{1}{c|}{} & \multicolumn{7}{c|}{ Decoding order ID } \\
\cline { 2 - 9 } & 1 & 2 & 3 & 4 & 5 & 6 & 7 & 8 \\
\hline u1 & u2-u3 & u2-u3 & u2-u3 & u2-u3 & u3-u2 & u3-u2 & u3-u2 & u3-u2 \\
\hline u2 & u1-u3 & u1-u3 & u3-u1 & u3-u1 & u1-u3 & u1-u3 & u3-u1 & u3-u1 \\
\hline u3 & u1-u2 & u2-u1 & u1-u2 & u2-u1 & u1-u2 & u2-u1 & u1-u2 & u2-u1 \\
\hline
\end{tabular}

Let $m, n$, and $p$ be the three users of the system. For any selected pair of users $(p, n)$, and for a given decoding order, their mutual SIC constraints fall in one of the three following categories of mutual SIC:

1) Users $p$ and $n$ did not decode the signal of user $m$ prior to decoding their respective signals. The users-decoding ID triplets $(p, n, \mathrm{ID})$ that fall into this category are: $(\mathrm{u} 1, \mathrm{u} 2,1)$, (u1,u2,2), (u2,u3,4), (u2,u3,8), (u1,u3,5), and (u1,u3,7).

2) User $p$ has been managed to decode the signal of user $m$ prior to decoding the signal of user $n$, while $n$ has not been managed to decode $s_{m}$ before proceeding with $s_{p}$. The corresponding ordered triplets $(p, n, \mathrm{ID})$ are: $(\mathrm{u} 1, \mathrm{u} 3,1)$, (u2,u3,2), (u2,u3,6), (u1,u3,3), (u3,u2,3), (u3,u2,7), 
$(\mathrm{u} 2, \mathrm{u} 1,3)(\mathrm{u} 2, \mathrm{u} 1,4),(\mathrm{u} 1, \mathrm{u} 2,5),(\mathrm{u} 1, \mathrm{u} 2,6),(\mathrm{u} 3, \mathrm{u} 1,6)$, and $(\mathrm{u} 3, \mathrm{u} 1,8)$.

3) Both users $p$ and $n$ have successfully decoded the signal of user $m$ prior to decoding each others signals. The corresponding triplets are: (u2,u3,1), (u2,u3,5), (u1,u3,2), (u1,u3,4), (u1,u2,7), and (u1,u2,8).

For every scenario, we start by identifying the interference sets $C_{p n}^{s_{n}}, C_{p n}^{s_{p}}, \mathcal{U}_{p}$ and $\mathcal{U}_{n}$, and then derive the mutual SIC conditions between $n$ and $p$. From Section III, we recall that $p \in \mathcal{C}_{p n}^{s_{n}}$ and $n \in \mathcal{C}_{p n}^{s_{p}}$.

Scenario 1: Users $p$ and $n$ did not decode $s_{m}$ before canceling each other's interference. In this case, $m$ is a common interfering signal to $p$ and $n$. Therefore $\mathcal{C}_{p n}^{s_{n}}=\{m, p\}$, $\mathcal{C}_{p n}^{s_{p}}=\{m, n\}$, and $\mathcal{U}_{n}=\mathcal{U}_{p}=\emptyset$. Using (6), we get the following condition at the level of user $p$ :

$$
\begin{gathered}
\left(h_{p, 1} h_{n, 2}-h_{p, 2} h_{n, 1}\right)\left[P_{n, 1}\left(P_{p, 2}+P_{m, 2}\right)-P_{n, 2}\left(P_{p, 1}+P_{m, 1}\right)\right] \\
>0 .
\end{gathered}
$$

The SIC condition at the level of user $n$ is simply obtained by interchanging $p$ and $n$ in the previous expression:

$$
\begin{gathered}
\left(h_{n, 1} h_{p, 2}-h_{n, 2} h_{p, 1}\right)\left[P_{p, 1}\left(P_{n, 2}+P_{m, 2}\right)-P_{p, 2}\left(P_{n, 1}+P_{m, 1}\right)\right] \\
>0 .
\end{gathered}
$$

By letting $H_{p n}=h_{p, 1} h_{n, 2}-h_{p, 2} h_{n, 1}$, the mutual SIC conditions can be written in the following form:

$$
\left\{\begin{array}{l}
H_{p n}\left[P_{n, 1}\left(P_{p, 2}+P_{m, 2}\right)-P_{n, 2}\left(P_{p, 1}+P_{m, 1}\right)\right]>0,(16) \\
H_{p n}\left[P_{p, 2}\left(P_{n, 1}+P_{m, 1}\right)-P_{p, 1}\left(P_{n, 2}+P_{m, 2}\right)\right]>0 .(17)
\end{array}\right.
$$

We note that, contrary to the two-user JT system, the SIC condition to remove $s_{p}$ at the level of $n$ is no longer the same as the SIC condition to cancel $s_{n}$ at the level of $p$. This means that it may happen that only one of the users succeeds in decoding the signal of the other one.

The PMCs for the removal of $s_{n}$ then $s_{m}$ at the level of user $p$ are respectively :

$$
\begin{gathered}
P_{n, 1} h_{p, 1}+P_{n, 2} h_{p, 2}>\left(P_{p, 1}+P_{m, 1}\right) h_{p, 1}+\left(P_{p, 2}+P_{m, 2}\right) h_{p, 2}, \\
P_{m, 1} h_{p, 1}+P_{m, 2} h_{p, 2}>P_{p, 1} h_{p, 1}+P_{p, 2} h_{p, 2} .
\end{gathered}
$$

The PMCs for the removal of $s_{p}$ then $s_{m}$ at the level of user $n$ are respectively :

$$
\begin{gathered}
P_{p, 1} h_{n, 1}+P_{p, 2} h_{n, 2}>\left(P_{n, 1}+P_{m, 1}\right) h_{n, 1}+\left(P_{n, 2}+P_{m, 2}\right) h_{n, 2}, \\
P_{m, 1} h_{n, 1}+P_{m, 2} h_{n, 2}>P_{n, 1} h_{n, 1}+P_{n, 2} h_{n, 2} .
\end{gathered}
$$

Scenario 2: User $p$ decoded $s_{m}$ and user $n$ did not decode $s_{m}$ before canceling their respective signals. The corresponding SIC condition at the level of user $p$ is (Cf. Appendix B):

$$
\begin{gathered}
{\left[P_{n, 1}\left(P_{p, 2}+P_{m, 2}\right)-P_{n, 2}\left(P_{p, 1}+P_{m, 1}\right)\right] H_{p n}} \\
+\left(P_{n, 1} h_{n, 1}+P_{n, 2} h_{n, 2}\right)\left[P_{m, 1} h_{p, 1}+P_{m, 2} h_{p, 2}\right]>0 .
\end{gathered}
$$

There is an additional positive term compared to (16). This means that the condition that must be satisfied to ensure SIC of $s_{n}$ at the level of $p$ is less stringent when $p$ has previously removed the message of user $m$. This result is shown here through calculation, but it is also intuitive, since removing the interference term of user $m$ enhances $S I N R_{p}^{s_{n}}$ compared to $S I N R_{n}^{s_{n}}$ in (4). On the other hand, this dissymmetry of the interfering user sets degrades the chances of $n$ to perform SIC of $s_{p}$ when compared to (17), as its $S I N R_{n}^{s_{p}}$ suffers from an interference that is not present in $S I N R_{p}^{s_{p}}$ in (5). This is also shown in the expression of the new SIC condition at the level of $n$ where an additional negative term is present. Indeed, let $B$ the expression of the SIC condition at the level of user $n$, its expression is given by (Cf. Appendix B):

$$
\begin{aligned}
B= & {\left[P_{p, 2}\left(P_{n, 1}+P_{m, 1}\right)-P_{p, 1}\left(P_{n, 2}+P_{m, 2}\right)\right] H_{p n} } \\
& -\left(P_{p, 1} h_{n, 1}+P_{p, 2} h_{n, 2}\right)\left[P_{m, 1} h_{p, 1}+P_{m, 2} h_{p, 2}\right]>0 .
\end{aligned}
$$

The PMCs for the removal of $s_{m}$ then $s_{n}$ at the level of user $p$ are respectively:

$P_{m, 1} h_{p, 1}+P_{m, 2} h_{p, 2}>\left(P_{p, 1}+P_{n, 1}\right) h_{p, 1}+\left(P_{p, 2}+P_{n, 2}\right) h_{p, 2}$,

$$
P_{n, 1} h_{p, 1}+P_{n, 2} h_{p, 2}>P_{p, 1} h_{p, 1}+P_{p, 2} h_{p, 2} .
$$

Also, the PMCs for the removal of $s_{p}$ then $s_{m}$ at the level of user $n$ are respectively:

$$
\begin{gathered}
P_{p, 1} h_{n, 1}+P_{p, 2} h_{n, 2}>\left(P_{n, 1}+P_{m, 1}\right) h_{n, 1}+\left(P_{n, 2}+P_{m, 2}\right) h_{n, 2} \\
P_{m, 1} h_{n, 1}+P_{m, 2} h_{n, 2}>P_{n, 1} h_{n, 1}+P_{n, 2} h_{n, 2} .
\end{gathered}
$$

Scenario 3: Users $p$ and $n$ decoded $s_{m}$ before canceling each other's interference. In this scenario, the conditions of mutual SIC between $p$ and $n$ are exactly the same as in the two-user system since the third user, $m$, is taken out of the equation for the two users. Therefore, the mutual SIC constraint is the same as (7):

$$
\left(h_{n, 1} h_{p, 2}-h_{n, 2} h_{p, 1}\right)\left[P_{p, 1} P_{n, 2}-P_{p, 2} P_{n, 1}\right]>0 .
$$

The signal of the third user $m$ must be the dominant one at both users $p$ and $n$. The PMCs of $p$ are as follows:

$$
\begin{gathered}
P_{m, 1} h_{p, 1}+P_{m, 2} h_{p, 2}>\left(P_{p, 1}+P_{n, 1}\right) h_{p, 1}+\left(P_{p, 2}+P_{n, 2}\right) h_{p, 2} \\
P_{n, 1} h_{p, 1}+P_{n, 2} h_{p, 2}>P_{p, 1} h_{p, 1}+P_{p, 2} h_{p, 2} .
\end{gathered}
$$

The PMCs for the removal of $s_{m}$ then $s_{p}$ at the level of user $n$ are:

$P_{m, 1} h_{n, 1}+P_{m, 2} h_{n, 2}>\left(P_{n, 1}+P_{p, 1}\right) h_{n, 1}+\left(P_{n, 2}+P_{p, 2}\right) h_{n, 2}$,

$$
P_{p, 1} h_{n, 1}+P_{p, 2} h_{n, 2}>P_{n, 1} h_{n, 1}+P_{n, 2} h_{n, 2} .
$$

In all eight configurations, the total power constraints are given by:

$$
\begin{aligned}
& P_{1,1}+P_{2,1}+P_{3,1} \leq P_{L_{1}}, \\
& P_{1,2}+P_{2,2}+P_{3,2} \leq P_{L_{2}} .
\end{aligned}
$$

To sum up, our proposed method, namely FullJTTripleMutSIC, serves all three users using joint transmission and seeks to achieve an interference-free NOMA cluster. For every channel realization, the method solves the problem of sum-rate maximization ( $\max R_{1}+R_{2}+R_{3}$, where $R_{i}$, $i=1,2,3$, are given in (2)) eight times with the corresponding PMCs and mutual SIC conditions for every corresponding configuration, while respecting the power limits imposed by the system in (18) and (19). The algorithm retains the results of the best performing configuration per channel realization. 
C. Enhancement over CellEdgeJT-CellCenterSIC: CellEdgeJTTripleMutSIC

Two major aspects differentiate FullJT-TripleMutSIC from CellEdgeJT-CellCenterSIC: the use of the mutual SIC procedure at all users, and the employment of JT to serve all users. However, the FullJT context is not necessary for achieving triple mutual SIC. Therefore, to assess separately the benefits of JT from those of mutual SIC, we propose enabling the use of the mutual SIC procedure in CellEdgeJT-CellCenterSIC when possible, calling it CellEdgeJT-TripleMutSIC. In this case, only the cell-edge user is served using JT, while all three users may cancel their mutual interferences.

CellEdgeJT-TripleMutSIC presents the advantage of using a triple mutual SIC over CellEdgeJT-CellCenterSIC, while FullJTTripleMutSIC presents the advantage of using a complete JT system over CellEdgeJT-TripleMutSIC. Moreover, the use of mutual SIC, and more precisely triple mutual SIC, allows the algorithm to reach a solution when the initial CellEdgeJTCellCenterSIC technique fails because the SIC conditions strongly depend on the channel conditions in (15): if the signs the channel differences don't match, SIC is not possible irrespectively of the power distribution. The PMCs and mutual SIC conditions are directly derived from the ones obtained in Section V-B by letting either $P_{p, 1}$ or $P_{p, 2}$ (resp. $P_{n, 1}$ or $P_{n 2}$, $P_{m 1}$ or $P_{m 2}$ ) be equal to zero, when a cell-center user $p$ (resp. $n, m)$ is concerned. The eight scenarios are then evaluated. However, because of the decrease in the degrees of freedom in the system (in terms of non-zero power variables), the chances of successive triple mutual SIC are lower with CellEdgeJTTripleMutSIC, compared to FullJT-TripleMutSIC. Therefore, the CellEdgeJT-TripleMutSIC technique first applies triple mutual SIC when possible. If no solution is found, CellEdgeJTCellCenterSIC is applied. If neither strategy leads to a solution, SIC is abandoned at all users levels, i.e. all the SIC and PMC constraints are relaxed and the rate maximization problem involves the sum rate of interference-full users. Their rates are given by :

$$
\begin{gathered}
R_{1}=\log _{2}\left(1+\frac{P_{1,1} h_{1,1}}{P_{3,1} h_{1,1}+\left(P_{3,2}+P_{2,2}\right) h_{1,2}+\sigma^{2}}\right), \\
R_{2}=\log _{2}\left(1+\frac{P_{2,2} h_{2,2}}{\left(P_{3,1}+P_{1,1}\right) h_{2,1}+P_{3,2} h_{2,2}+\sigma^{2}}\right), \\
R_{3}=\log _{2}\left(1+\frac{P_{3,1} h_{3,1}+P_{3,2} h_{3,2}}{P_{1,1} h_{3,1}+P_{2,2} h_{3,2}+\sigma^{2}}\right) .
\end{gathered}
$$

\section{On successful SIC in FullJT-TripleMutSIC and CellEdgeJT- TripleMutSIC}

Achieving a complete mutual SIC in three-user NOMA clusters using two serving antennas is no longer guaranteed as it was the case for DPS-MutSIC and JT-MutSIC in twouser clusters. In such situations, it is possible to evaluate the alternatives where a smaller number of users operate in mutual SIC while the rest may benefit from single SIC or not. However, this is out of the scope of this paper as we are only interested in the cases of full triple mutual SIC. In other words, not all three methods are guaranteed to yield a successful triple mutual SIC implementation for all simulations. Therefore, it is logical to assume that the three considered methods do not show the same level of success rate for triple mutual SIC. An analysis is conducted in Section VI to get an insight on the statistics of triple mutual SIC occurrences.

\section{Vi. Performance Evaluation}

Simulations are conducted to evaluate the performance of the presented scenarios and techniques, under the following practical conditions: The outer cell radius of each hexagonal cell is $R_{d}=500 \mathrm{~m}$. The penetration depth of the user 3 zone is of $30 \mathrm{~m}$ in each cell (Cf. Fig. 1). Three out of the four RRHs (per cell) are spread across the cell, uniformly positioned on a circle of radius $2 R_{d} / 3$, while the fourth is located at each cell center. Users are independently positioned, their positions being randomly generated with a uniform probability distribution over their respective regions. The transmission channel model includes a distance-dependent path-loss of decay factor 3.76, and a zero-mean lognormal shadowing with an $8 \mathrm{~dB}$ variance. The considered sub-band bandwidth is $B=156.250 \mathrm{kHz}$ (equivalent to a total bandwidth of $10 \mathrm{MHz}$ subdivided into 64 sub-bands). The power spectral density of the additive background white noise is $N_{0}=4 \cdot 10^{-18} \mathrm{~mW} / \mathrm{Hz}$, and the noise power on each sub-band is $\sigma^{2}=N_{0} B$. The power limit constraints over the serving antennas $r_{1}$ and $r_{2}$ are varied such that the total available system power $P_{L}$ (excluding other nonserving RRHs) remains constant throughout the simulations. Unless specified otherwise, the total power $P_{L}=P_{L_{1}}+P_{L_{2}}$ is $4 \mathrm{~W}$. MATLAB software is used to generate the numerical results and fmincon from the optimization toolbox is used to solve the optimization problems in each technique.

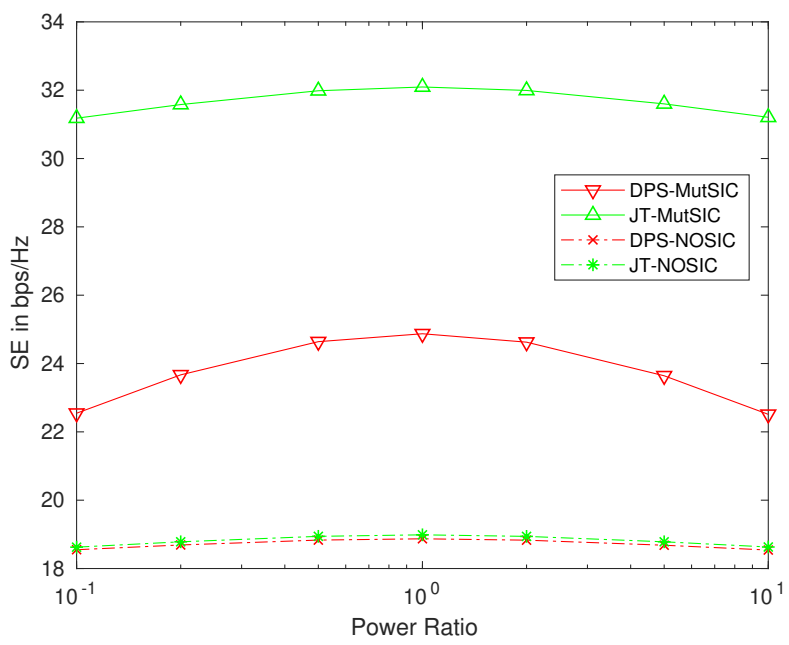

Fig. 2: Spectral Efficiency of a two-user system as a function of $P_{L_{1}} / P_{L_{2}}$

In Fig.2, the system Spectral Efficiency (SE) for the different two-user strategies are presented as a function of the antennas power limit ratio $P_{L_{1}} / P_{L_{2}}$. Although antenna power limits of different cells are not usually linked, the chosen representation provides a useful analysis, for network planning, of the best power distribution between the antennas. A first noticeable property is the shape of the curves: all the techniques seem to 
reach their performance peak at the unity power ratio, implying that the system better handles different user distributions when $P_{L_{1}}=P_{L_{2}}$. It should be pointed out, though, that this observation is only true on an average basis, i.e. the optimal power ratio is not necessarily one for every single channel realization.

At the common performance peak $\left(P_{L_{1}}=P_{L_{2}}=2 \mathrm{~W}\right)$, an important SE gap between MutSIC and No-SIC algorithms is observed for both the JT and DPS cases. SE gains of $13.1 \mathrm{bps} / \mathrm{Hz}(69 \%$ increase) and $6 \mathrm{bps} / \mathrm{Hz}$ (32\% increase) are achieved between JT-MutSIC and JT-NoSIC, and between DPSMutSIC and DPS-NoSIC respectively. This clearly showcases the superiority of the mutual SIC procedure with respect to the common practice of automatically assigning the users to their best antennas which is implicitly done in the No-SIC algorithms as discussed hereafter.

The JT algorithms dominate their DPS counterparts in both mutual SIC and NoSIC scenarios. However, the performance gap between DPS-NoSIC and JT-NoSIC is nearly imperceptible. To understand this behavior, we first recall the four possible DPS-NoSIC scenarios of Section IV-A2, where users can be served either by the same antenna or by different ones. Any of these four cases can be derived from the JT version of this algorithm by simply setting the appropriate power variables to zero. Once again, JT encompasses all the different DPS scenarios into a broader one. The simulation results reveal that the power allocation scheme that maximizes the total rate for DPS-NoSIC, almost always resides in allocating to the user with the best channel gain the entire power $P_{L}$ of the serving RRH. The signal of the second user is switched off, whether or not it is served by the same RRH, avoiding thereby the interference that would be caused by that user. In such cases, the enhancement brought by the JT scenario is in the addition of a new signal coming from the second antenna that enhances the reception quality of the user, and thus its rate as well as the total system rate. The problem occurs when the user rate vs. SNR (signal-to-noise ratio) curve is already at a saturation point. Therefore, the increase in the power level translates into a marginal rate enhancement. In contrast, the more equitable power distribution, that takes place in DPS-MutSIC between users, renders the working point of every user rather far from the saturation region of their rate vs. SNR curves. This effect is reminiscent of the waterfilling algorithm where maximizing the total rate is done through shifting some of the available power away from the best link, towards another, rather than focusing the whole power on the best link. The only difference here is that instead of having multiple sub-bands allocated to one user, the same sub-band is allocated to two different users at the same time. In this regard, the effect of the mutual SIC procedure is virtually doubling the bandwidth of the system without adding interference. Not only does this achieve a much greater fairness and more important sum rates, but it also yields a significant rate improvement when moving from DPS-MutSIC to JT-MutSIC, as shown in Fig. 2.

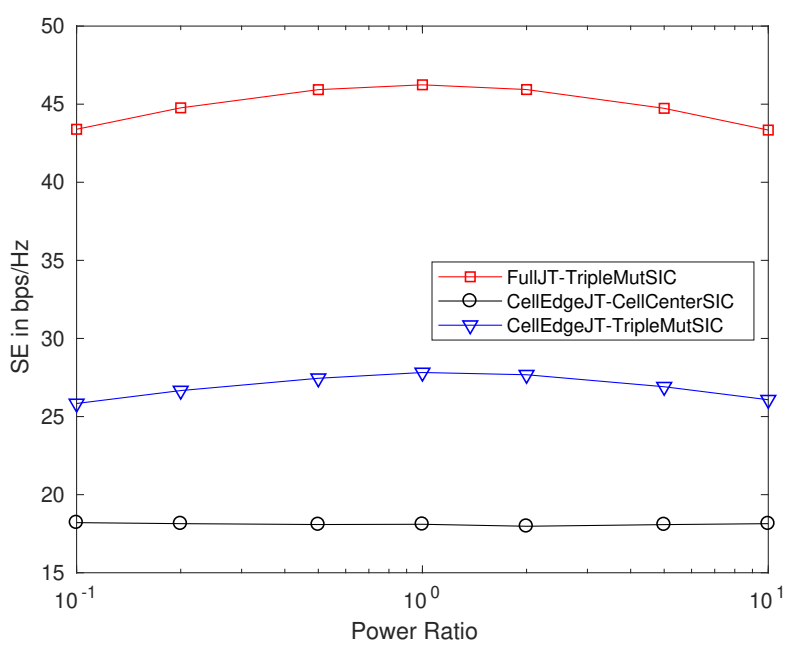

Fig. 3: Comparison of the rate maximization procedures for a three-user system

The performance of the discussed methods for three-user clusters are presented in Fig. 3. As stated earlier, a complete mutual SIC procedure is no longer guaranteed in three-user systems, and different techniques lead to different success rates for triple mutual SIC. For our setup, a statistical analysis of the obtained results yields $95 \%$ chances of successful mutual SIC in FullJT-TripleMutSIC and $46 \%$ in CellEdgeJTTripleMutSIC. The analysis also shows that even the easier single SIC conditions in CellEdgeJT-CellCenterSIC are not always feasible, with $44 \%$ success rate for SIC of the signal of user $3, s_{3}$ at the level of user 1 and user 2 .

Comparing CellEdgeJT-CellCenterSIC and CellEdgeJTTripleMutSIC showcases the enhancements brought by adopting the triple mutual SIC strategy (18.2 bps/Hz vs. $27.8 \mathrm{bps} / \mathrm{Hz}$ at the peak). Indeed, the rate gain is entirely due to the use of the triple mutual SIC procedure, as no change is carried to the system configuration: in both cases, user 3 is served with JT and users 1 and 2 are served by a single antenna in DPS. This shows that the occurrence of triple mutual SIC is not exclusively bound to a full JT NOMA cluster, and it highlights the ability of triple mutual SIC to increase the total throughput without requiring any technical change in the system. On the other hand, comparing FullJT-TripleMutSIC and CellEdgeJTTripleMutSIC sheds the light on the importance of a fully JT-based system in enhancing the throughput. This time, the use of JT to serve every user distinguishes FullJT-TripleMutSIC from CellEdgeJT-TripleMutSIC. As in Fig.2, the rate gain due to JT towards DPS is magnified by triple mutual SIC where a rate gain of $18.4 \mathrm{bps} / \mathrm{Hz}$ is achieved (66\% increase).

TABLE III: Jain fairness measure for three-user systems for $P_{L_{1}} / P_{L_{2}}=1$

\begin{tabular}{|c|c|}
\cline { 2 - 2 } \multicolumn{1}{c|}{} & Jain fairness \\
\hline FullJT-TripleMutSIC & 0.97 \\
\hline CellEdgeJT-CellCenterSIC & 0.40 \\
\hline CellEdgeJT-TripleMutSIC & 0.62 \\
\hline
\end{tabular}

The fairness measure of the three-user techniques is provided 
in Table III for a unit power ratio $\left(P_{L_{1}} / P_{L_{2}}=1\right)$. The Jain fairness index is used [29]. This index is upper bounded by 1 for absolute fairness scenarios (i.e. all users achieve the same rate on average), and lower bounded by $1 / 3$ which corresponds to the worst case scenario (i.e. a single user is holding all of the system throughput). The fairness index achieved by FullJT-TripleMutSIC approaches the upper bound whereas the CellEdgeJT-CellCenterSIC technique has a poor fairness index (0.40). This shows that not only does FullJT-TripleMutSIC perform best with regards to SE, but it also achieves the highest values of fairness among users. Thanks to the mutual SIC procedure, FullJT-TripleMutSIC achieves a higher system throughput through a fairer distribution of the available power to the users. To better showcase this behavior, the individual rates of users are presented for both FullJT-TripleMutSIC and CellEdgeJT-CellCenterSIC as a function of the total system power in Fig. 4. Instead of showing the average individual rate achieved by each user, the averages of the minimum, maximum and middle rates achieved in every simulation are put forward, in order to better emphasize the throughput disparity for the different methods.

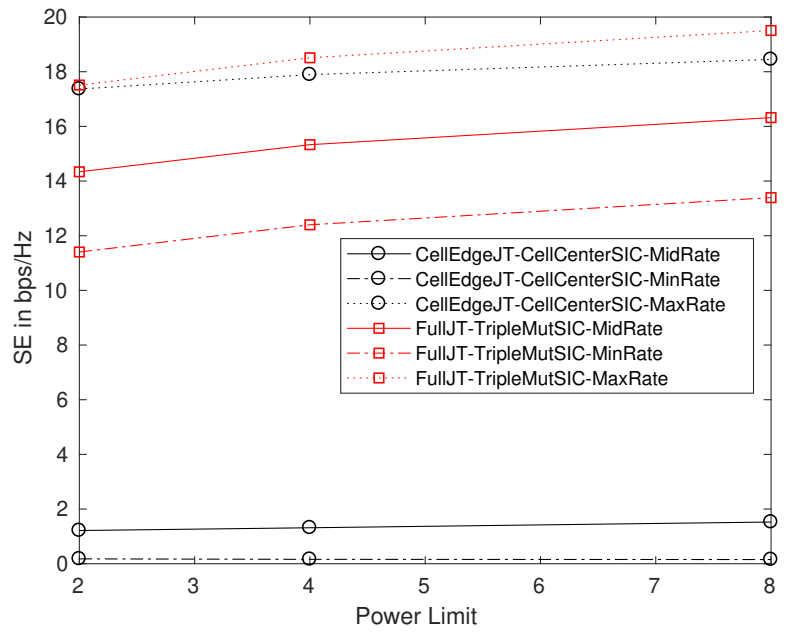

Fig. 4: Minimum, maximum and middle individual user rates as a function of the system power for a power ratio equal to one in a three-user system

It can be seen from Fig. 4 that most of the throughput achieved by CellEdgeJT-CellCenterSIC comes from the highest rate user. Indeed, for a total system power of $8 \mathrm{~W}$, the minimum and middle rate users account for only $8.3 \%$ of the total throughput, compared to the $60 \%$ for FullJT-TripleMutSIC. The rate distribution in FullJT-TripleMutSIC is much fairer, each user actively contributing to the system throughput.

In Fig. 5, the best performing approach for two and threeuser clusters are compared in the same conditions of power ratios and total system power. Also, to allow a fair comparison, the user deployment is kept unvaried for the two initial users: for every channel realization, users 1 and 2 are randomly deployed according to the system model in Fig. 1, and the third user is added to the system without affecting the initial distribution of the two other users. Even under these conditions, the rate gain provided by the third user accounts to a $44 \%$ increase in SE, for a power ratio equal to one when $P_{L}=4 \mathrm{~W}$. This significant increase is not only due to the exploitation of the added diversity by the third user. In fact, being able to serve a third user without causing interference - which is the core of triple mutual SIC - is equivalent to adding to the system an additional virtual sub-band for exploitation. This was the case for JT-MutSIC compared to JT-NoSIC, and it is also the case of FullJT-TripleMutSIC in comparison with JTMutSIC. Also, this result contrasts with the general knowledge inherent to classical single-SIC NOMA systems, such as in [30], where it is shown that the performance gain of three vs. two collocated users per sub-band and powered by the same antenna is rather minor. With a judicious NOMA-DAS employing mutual SIC, the number of users per cluster could be efficiently extended to the limit that can be allowed by both the SIC complexity constraint at receivers, and the large but yet limited computational power available at the BBU for scheduling. Due to the exponential increase of the scheduling complexity with the cluster size, the best trade-off is usually attained for two or three-user clusters.

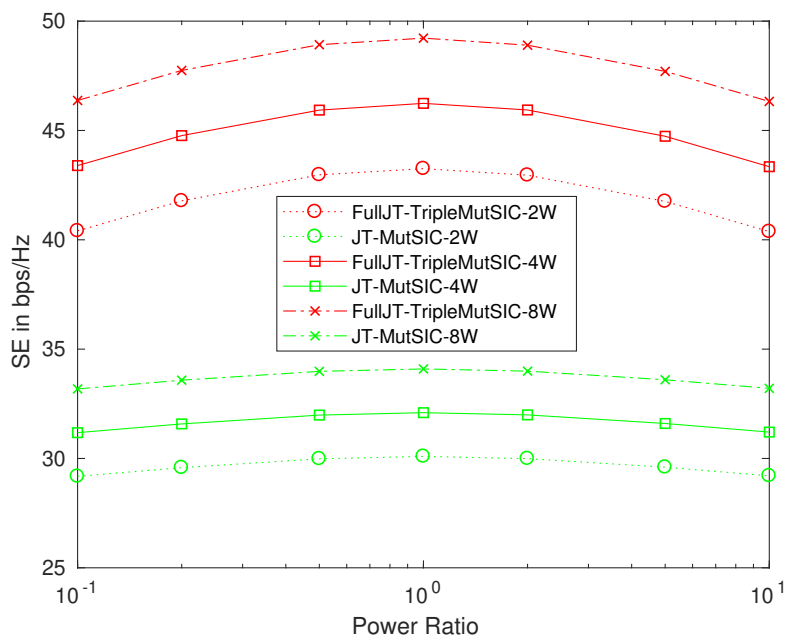

Fig. 5: Comparison of the best performing scenario for 2-user vs. 3-user clusters, for $P_{L}=2,4$ and $8 \mathrm{~W}$

Comparing the rates for different power values, it appears that a linear increase in the throughput occurs for a geometric progression in the total power. This is to be expected given the logarithmic relation between the serving power and the rate (Cf. equations (1) and (2)). Furthermore, it can be observed that rate curves for different power limits are parallel which reinforces the idea that maximum throughput is achieved, on average, for unit power ratios.

As a conclusion, FullJT-TripleMutSIC is by far the best performing technique. Even though particularly restrictive measures on antenna selection were set in our study by limiting the serving antenna choices to $r_{1}$ and $r_{2}$ in the configuration of Fig.1, an important success rate to establish triple mutual SIC was observed with $95 \%$ chances. Furthermore, it is expected that taking advantage of the spatial diversity of each cell by fully exploiting the DAS system would yield even higher percentages 
of triple mutual SICs. Moreover, the frequency diversity of the system was not taken into account in the study, since a flat fading channel was assumed in our simulations. In fact, having seen the efficiency of triple mutual SIC, a new way of user-clustering can be envisioned in which the selection of user 1 and user 2, RRHs $r_{1}$ and $r_{2}$, and the sub-band, are based on the cell-edge user, in order to guarantee a triple mutual SIC implementation.

\section{CONCLUSION}

In this study, we tackled the combination of NOMA with CoMP systems to enhance cell-edge user experience as well as the global system performance. We first explored the conditions for a mutual SIC procedure for a general NOMA cluster with two coordinated antennas. The mutual SIC procedure was then applied to two-user and three-user clusters in both DPS and JT. Important performance enhancements were shown in the system throughput and the user fairness which validate the potential of this technology in reaching current and future challenges imposed by $5 \mathrm{G}$ and beyond systems. 


\section{APPENDIX A}

\section{DERIVATION OF CONDITIONS FOR MUTUAL SIC}

The SINR condition for the decoding of $s_{n}$ at the level of $p$ is: $S I N R_{p}^{s_{n}}>S I N R_{n}^{s_{n}}$. By subtracting $S I N R_{n}^{s_{n}}$ from $S I N R_{p}^{s_{n}}$ we get:

$$
\operatorname{SINR}_{p}^{s_{n}}-\operatorname{SINR}_{n}^{s_{n}}=\frac{P_{n, 1} h_{p, 1}+P_{n, 2} h_{p, 2}}{\sum_{i \in \mathcal{I}_{p n}^{s_{n}}}\left(P_{i, 1} h_{p, 1}+P_{i, 2} h_{p, 2}\right)+\sigma^{2}}-\frac{P_{n, 1} h_{n, 1}+P_{n, 2} h_{n, 2}}{\sum_{i \in \mathcal{I}_{p n}^{s_{n}}}\left(P_{i, 1} h_{n, 1}+P_{i, 2} h_{n, 2}\right)+\sigma^{2}}>0,
$$

which leads to

$A=\left(P_{n, 1} h_{p, 1}+P_{n, 2} h_{p, 2}\right)\left[\sum_{i \in \mathcal{I}_{n}^{s_{n}}}\left(P_{i, 1} h_{n, 1}+P_{i, 2} h_{n, 2}\right)+\sigma^{2}\right]-\left(P_{n, 1} h_{n, 1}+P_{n, 2} h_{n, 2}\right)\left[\sum_{i \in \mathcal{I}_{p}^{s_{n}}}\left(P_{i, 1} h_{p, 1}+P_{i, 2} h_{p, 2}\right)+\sigma^{2}\right]>0$,

where $A$ is the numerator of $S I N R_{p}^{s_{n}}-S I N R_{n}^{s_{n}}$, its expression can be further rearranged as:

$$
\begin{aligned}
A & =h_{n, 1} h_{p, 1} P_{n, 1}\left[\sum_{i \in \mathcal{I}_{n}^{s_{n}}} P_{i, 1}-\sum_{i \in \mathcal{I}_{p}^{s_{n}}} P_{i, 1}\right]+h_{n, 2} h_{p, 2} P_{n, 2}\left[\sum_{i \in \mathcal{I}_{n}^{s_{n}}} P_{i, 2}-\sum_{i \in \mathcal{I}_{p}^{s_{n}}} P_{i, 2}\right]+\sigma^{2}\left[P_{n, 1}\left(h_{p, 1}-h_{n, 1}\right)+P_{n, 2}\left(h_{p, 2}-h_{n, 2}\right)\right] \\
& +\underbrace{h_{p, 1} h_{n, 2}\left[P_{n, 1} \sum_{i \in \mathcal{I}_{n}^{s_{n}}} P_{i, 2}-P_{n, 2} \sum_{i \in \mathcal{I}_{p}^{s_{n}}} P_{i, 1}\right]+h_{p, 2} h_{n, 1}\left[P_{n, 2} \sum_{i \in \mathcal{I}_{n}^{s_{n}}} P_{i, 1}-P_{n, 1} \sum_{i \in \mathcal{I}_{p}^{s_{n}}} P_{i, 2}\right]}_{B} .
\end{aligned}
$$

By detailing $B$, we get:

$$
\begin{aligned}
B & =h_{p, 1} h_{n, 2}\left[P_{n, 1}\left(\sum_{i \in \mathcal{C}_{p n}^{s n}} P_{i, 2}+\sum_{i \in \mathcal{U}_{n}} P_{i, 2}\right)-P_{n, 2}\left(\sum_{i \in \mathcal{C}_{p n}^{s_{n}}} P_{i, 1}+\sum_{i \in \mathcal{U}_{p}} P_{i, 1}\right)\right] \\
& +h_{p, 2} h_{n, 1}\left[P_{n, 2}\left(\sum_{i \in \mathcal{C}_{p n}^{s_{n}}} P_{i, 1}+\sum_{i \in \mathcal{U}_{n}} P_{i, 1}\right)-P_{n, 1}\left(\sum_{i \in \mathcal{C}_{p n}^{s_{n}}} P_{i, 2}+\sum_{i \in \mathcal{U}_{n}} P_{i, 2}\right)\right], \\
B & =\left(h_{p, 1} h_{n, 2}-h_{p, 2} h_{n, 1}\right)\left[P_{n, 1} \sum_{i \in \mathcal{C}_{p n}^{s_{n}}} P_{i, 2}-P_{n, 2} \sum_{i \in \mathcal{C}_{p n}^{s_{n}}} P_{i, 1}\right]+h_{p, 1} h_{n, 2}\left[P_{n, 1} \sum_{i \in \mathcal{U}_{n}} P_{i, 2}-P_{n, 2} \sum_{i \in \mathcal{U}_{p}} P_{i, 1}\right] \\
& +h_{p, 2} h_{n, 1}\left[P_{n, 2} \sum_{i \in \mathcal{U}_{n}} P_{i, 1}-P_{n, 1} \sum_{i \in \mathcal{U}_{p}} P_{i, 2}\right] .
\end{aligned}
$$

In practical interference-limited systems, the background noise is negligible compared to the interfering signals [31], [32], i.e. $\sigma^{2}<<P_{k^{\prime}, r} h_{k, r}, \forall\left(k, k^{\prime}\right) \in \mathcal{K}^{2}, \forall r \in\left\{r_{1}, r_{2}\right\}$. Replacing $B$ by its expression in $A$, we get the final expression of the SIC condition for the decoding of $s_{n}$ at the level of user $p$ :

$$
\begin{aligned}
A & =h_{n, 1} h_{p, 1} P_{n, 1}\left[\sum_{i \in \mathcal{U}_{n}} P_{i, 1}-\sum_{i \in \mathcal{U}_{p}} P_{i, 1}\right]+h_{n, 2} h_{p, 2} P_{n, 2}\left[\sum_{i \in \mathcal{U}_{n}} P_{i, 2}-\sum_{i \in \mathcal{U}_{p}} P_{i, 2}\right]+h_{p, 1} h_{n, 2}\left[P_{n, 1} \sum_{i \in \mathcal{U}_{n}} P_{i, 2}-P_{n, 2} \sum_{i \in \mathcal{U}_{p}} P_{i, 1}\right] \\
& +h_{p, 2} h_{n, 1}\left[P_{n, 2} \sum_{i \in \mathcal{U}_{n}} P_{i, 1}-P_{n, 1} \sum_{i \in \mathcal{U}_{p}} P_{i, 2}\right]+\left(h_{p, 1} h_{n, 2}-h_{p, 2} h_{n, 1}\right)\left[P_{n, 1} \sum_{i \in \mathcal{C}_{p n}^{s_{n}}} P_{i, 2}-P_{n, 2} \sum_{i \in \mathcal{C}_{p n}^{s_{n}}} P_{i, 1}\right]>0 .
\end{aligned}
$$




\section{APPENDIX B}

\section{Detailed Calculation of THE SIC Constraints For SCENARIO 2 IN SECTION V-B}

User $p$ decoded $s_{m}$ and user $n$ did not decode $s_{m}$ before canceling their respective signals. In this scenario, $m$ only affects the interfering set of user $n$, therefore we have $\mathcal{U}_{n}=$ $\{m\}, \mathcal{U}_{p}=\emptyset, \mathcal{C}_{p n}^{s_{n}}=\{p\}, \mathcal{C}_{p n}^{s_{p}}=\{n\}$. Let $A$ be the expression of the SIC condition at the level of user $p$. Using (6), we have:

$$
\begin{gathered}
A=h_{n, 1} h_{p, 1} P_{n, 1} P_{m, 1}+h_{n, 2} h_{p, 2} P_{n, 2} P_{m, 2} \\
+h_{p, 1} h_{n, 2} P_{n, 1} P_{m, 2}+h_{p, 2} h_{n, 1} P_{n, 2} P_{m, 1} \\
+\left(h_{p, 1} h_{n, 2}-h_{p, 2} h_{n, 1}\right)\left(P_{n, 1} P_{p, 2}-P_{n, 2} P_{p, 1}\right)>0 .
\end{gathered}
$$

By adding and subtracting $h_{p, 2} h_{n, 1} P_{n, 1} P_{m, 2}$ and $h_{p, 1} h_{n, 2} P_{n, 2} P_{m, 1}$ to $A$, we get:

$$
\begin{aligned}
A & =h_{n, 1} h_{p, 1} P_{n, 1} P_{m, 1}+h_{n, 2} h_{p, 2} P_{n, 2} P_{m, 2} \\
& +h_{p, 2} h_{n, 1} P_{n, 1} P_{m, 2}+h_{p, 1} h_{n, 2} P_{n, 2} P_{m, 1} \\
& -h_{p, 2} h_{n, 1} P_{n, 1} P_{m, 2}-h_{p, 1} h_{n, 2} P_{n, 2} P_{m, 1} \\
& +h_{p, 1} h_{n, 2} P_{n, 1} P_{m, 2}+h_{p, 2} h_{n, 1} P_{n, 2} P_{m, 1} \\
& +\left(h_{p, 1} h_{n, 2}-h_{p, 2} h_{n, 1}\right)\left(P_{n, 1} P_{p, 2}-P_{n, 2} P_{p, 1}\right) .
\end{aligned}
$$

After taking out common factors in the first two terms and in the last three, and after simplification $A$, becomes:

$$
\begin{aligned}
A & =\left[P_{n, 1}\left(P_{p, 2}+P_{m, 2}\right)-P_{n, 2}\left(P_{p, 1}+P_{m, 1}\right)\right] H_{p n} \\
& +\left(P_{n, 1} h_{n, 1}+P_{n, 2} h_{n, 2}\right)\left[P_{m, 1} h_{p, 1}+P_{m, 2} h_{p, 2}\right]>0 .
\end{aligned}
$$

To obtain the SIC conditions at the level of user $n, n$ and $p$ must be interchanged in the initial SIC condition in (6) before any simplification. By letting $B$ be the expression of the SIC condition we get:

$$
\begin{gathered}
B=h_{p, 1} h_{n, 1} P_{p, 1}\left[\sum_{i \in \mathcal{U}_{p}} P_{i, 1}-\sum_{i \in \mathcal{U}_{n}} P_{i, 1}\right] \\
+h_{p, 2} h_{n, 2} P_{p, 2}\left[\sum_{i \in \mathcal{U}_{p}} P_{i, 2}-\sum_{i \in \mathcal{U}_{n}} P_{i, 2}\right] \\
+h_{n, 1} h_{p, 2}\left[P_{p, 1} \sum_{i \in \mathcal{U}_{p}} P_{i, 2}-P_{p, 2} \sum_{i \in \mathcal{U}_{n}} P_{i, 1}\right] \\
+h_{n, 2} h_{p, 1}\left[P_{p, 2} \sum_{i \in \mathcal{U}_{p}} P_{i, 1}-P_{p, 1} \sum_{i \in \mathcal{U}_{n}} P_{i, 2}\right] \\
+\left(h_{n, 1} h_{p, 2}-h_{n, 2} h_{p, 1}\right)\left[P_{p, 1} \sum_{i \in \mathcal{C}_{p n}^{s_{p}}} P_{i, 2}-P_{p, 2} \sum_{i \in \mathcal{C}_{p n}^{s_{p}}} P_{i, 1}\right]>0 .
\end{gathered}
$$

Replacing $\mathcal{U}_{n}$ by $\{m\}, \mathcal{U}_{p}$ by $\emptyset$, and $\mathcal{C}_{p n}^{s_{p}}$ by $\{n\}, B$ becomes:

$$
\begin{gathered}
B=-h_{p, 1} h_{n, 1} P_{p, 1} P_{m, 1}-h_{p, 2} h_{n, 2} P_{p, 2} P_{m, 2} \\
-h_{n, 1} h_{p, 2} P_{p, 2} P_{m, 1}-h_{n, 2} h_{p, 1} P_{p, 1} P_{m, 2} \\
+\left(h_{n, 1} h_{p, 2}-h_{n, 2} h_{p, 1}\right)\left(P_{p, 1} P_{n, 2}-P_{p, 2} P_{n, 1}\right) .
\end{gathered}
$$

By adding and subtracting $h_{n, 2} h_{p, 1} P_{p, 2} P_{m, 1} \quad$ and $h_{n, 1} h_{p, 2} P_{p, 1} P_{m, 2}$ to $B$, we get:

$$
\begin{gathered}
B=h_{n, 2} h_{p, 1} P_{p, 2} P_{m, 1}+h_{n, 1} h_{p, 2} P_{p, 1} P_{m, 2} \\
-h_{n, 1} h_{p, 2} P_{p, 2} P_{m, 1}-h_{n, 2} h_{p, 1} P_{p, 1} P_{m, 2} \\
-h_{p, 1} h_{n, 1} P_{p, 1} P_{m, 1}-h_{p, 2} h_{n, 2} P_{p, 2} P_{m, 2} \\
-h_{n, 2} h_{p, 1} P_{p, 2} P_{m, 1}-h_{n, 1} h_{p, 2} P_{p, 1} P_{m, 2} \\
+\left(h_{n, 1} h_{p, 2}-h_{n, 2} h_{p, 1}\right)\left(P_{p, 1} P_{n, 2}-P_{p, 2} P_{n, 1}\right) .
\end{gathered}
$$

Combining the first two terms together and the third and forth ones together $B$ becomes:

$$
\begin{aligned}
B & =\left(h_{n, 2} h_{p, 1}-h_{n, 1} h_{p, 2}\right) P_{p, 2} P_{m, 1} \\
& +\left(h_{n, 1} h_{p, 2}-h_{n, 2} h_{p, 1}\right) P_{p, 1} P_{m, 2} \\
& -h_{p, 1} P_{m, 1}\left(h_{n, 1} P_{p, 1}+h_{n, 2} P_{p, 2}\right) \\
& -h_{p, 2} P_{m, 2}\left(h_{n, 2} P_{p, 2}+h_{n, 1} P_{p, 1}\right) \\
& +\left(h_{n, 1} h_{p, 2}-h_{n, 2} h_{p, 1}\right)\left(P_{p, 1} P_{n, 2}-P_{p, 2} P_{n, 1}\right) .
\end{aligned}
$$

Finally, grouping the common factors leads to the following expression:

$$
\begin{aligned}
B= & {\left[P_{p, 2}\left(P_{n, 1}+P_{m, 1}\right)-P_{p, 1}\left(P_{n, 2}+P_{m, 2}\right)\right] H_{p n} } \\
& -\left(h_{n, 1} P_{p, 1}+h_{n, 2} P_{p, 2}\right)\left[h_{p, 1} P_{m, 1}+h_{p, 2} P_{m, 2}\right] .
\end{aligned}
$$

\section{REFERENCES}

[1] Cisco, "Cisco Visual Networking Index: Forecast and Trends, 2017-2022," Nov. 2018.

[2] C. He, G. Y. Li, F. Zheng, and X. You, "Energy-Efficient Resource Allocation in OFDM Systems With Distributed Antennas," IEEE Trans. on Veh. Technol., vol. 63, no. 3, pp. 1223-1231, Mar. 2014.

[3] Q. Yu, Y. Li, W. Xiang, W. Meng, and W. Tang, "Power Allocation for Distributed Antenna Systems in Frequency-Selective Fading Channels," IEEE Trans. on Commun., vol. 64, no. 1, pp. 212-222, Jan. 2016.

[4] M. J. Youssef, J. Farah, C. Abdel Nour, and C. Douillard, "Resource Allocation for Mixed Traffic Types in Distributed Antenna Systems Using NOMA," in 2018 IEEE 77th Veh. Technol. Conf. (VTC fall), Aug. 2018, pp. $1-5$

[5] X. Gu, X. Ji, Z. Ding, W. Wu, and M. Peng, "Outage Probability Analysis of Non-Orthogonal Multiple Access in Cloud Radio Access Networks," IEEE Commun. Lett., vol. 22, no. 1, pp. 149-152, Jan. 2018.

[6] D. Boviz, C. S. Chen, and S. Yang, "Effective Design of Multi-User Reception and Fronthaul Rate Allocation in 5G Cloud RAN," IEEE J. on Sel. Areas in Commun., vol. 35, no. 8, pp. 1825-1836, Aug. 2017.

[7] F. Liu, E. Bala, E. Erkip, M. C. Beluri, and R. Yang, "Small-Cell Traffic Balancing Over Licensed and Unlicensed Bands," IEEE Trans. on Veh. Technol., vol. 64, no. 12, pp. 5850-5865, Dec. 2015.

[8] C. Li, J. Zhang, M. Haenggi, and K. B. Letaief, "User-Centric Intercell Interference Nulling for Downlink Small Cell Networks," IEEE Trans. on Commun., vol. 63, no. 4, pp. 1419-1431, Apr. 2015.

[9] O. Onireti, A. Zoha, J. Moysen, A. Imran, L. Giupponi, M. A. Imran, and A. Abu-Dayya, "A Cell Outage Management Framework for Dense Heterogeneous Networks," IEEE Trans. on Veh. Technol., vol. 65, no. 4, pp. 2097-2113, Apr. 2016.

[10] C. M. Moreira, G. Kaddoum, and E. Bou-Harb, "Cross-Layer Authentication Protocol Design for Ultra-Dense 5G HetNets," in 2018 IEEE Int. Conf. on Commun. (ICC), May. 2018, pp. 1-7.

[11] L. Dai, B. Wang, Y. Yuan, S. Han, C. I, and Z. Wang, "Non-Orthogonal Multiple Access for 5G: Solutions, Challenges, Opportunities, and Future Research Trends,," IEEE Commun. Mag., vol. 53, no. 9, pp. 74-81, Sept. 2015.

[12] M. Hojeij, C. Abdel Nour, J. Farah, and C. Douillard, "Waterfilling-Based Proportional Fairness Scheduler for Downlink Non-Orthogonal Multiple Access," IEEE Wireless Commun. Lett., vol. 6, no. 2, pp. 230-233, Apr. 2017.

[13] Y. Saito, Y. Kishiyama, A. Benjebbour, T. Nakamura, A. Li, and K. Higuchi, "Non-Orthogonal Multiple Access (NOMA) for Cellular Future Radio Access," in 2013 IEEE 77th Veh. Technol. Conf. (VTC Spring), June 2013, pp. 1-5.

[14] J. Farah, E. Sfeir, C. Abdel Nour, and C. Douillard, "New Resource Allocation Techniques for Base Station Power Reduction in Orthogonal and Non-Orthogonal Multiplexing Systems," in 2017 IEEE Int. Conf. on Commun. Workshops (ICC Workshops), May 2017, pp. 618-624.

[15] Z. Ding, Z. Yang, P. Fan, and H. V. Poor, "On the Performance of NonOrthogonal Multiple Access in 5G Systems with Randomly Deployed Users," IEEE Signal Process. Lett., vol. 21, no. 12, pp. 1501-1505, Dec. 2014. 
[16] M. S. Ali, H. Tabassum, and E. Hossain, "Dynamic User Clustering and Power Allocation for Uplink and Downlink Non-Orthogonal Multiple Access (NOMA) Systems," IEEE Access, vol. 4, pp. 6325-6343, 2016.

[17] 3GPP, "Mobile Broadband Innovation path to 4G: Release 9,10 and Beyond," 3rd Generation Partnership Project (3GPP), TR, Feb. 2010.

[18] 3GPP, "3GPP TR 36.819 Technical Specification Group Radio Access Network; Coordinated Multi-Point Operation for LTE Physical Layer Aspects," 2011.

[19] M. D. Michelle and J. S. Harrison, "CoMP (1): CoMP Types - CS, CB, JT and DPS," Aug. 2014. [Online]. Available: www.netmanias.com/en/post/blog/6558/comp-lte-lte-a/comp- 1-comptypes-cs-cb-jt-and-dps

[20] E. Pateromichelakis, M. Shariat, A. u. Quddus, and R. Tafazolli, "On the Evolution of Multi-Cell Scheduling in 3GPP LTE / LTE-A," IEEE Commun. Surveys Tuts, vol. 15, no. 2, pp. 701-717, Second 2013.

[21] A. Beylerian and T. Ohtsuki, "Coordinated Non-Orthogonal Multiple Access (CO-NOMA)," in 2016 IEEE Global Commun. Conf. (GLOBECOM), Dec. 2016, pp. 1-5.

[22] M. S. Ali, E. Hossain, and D. I. Kim, "Coordinated Multipoint Transmission in Downlink Multi-Cell NOMA Systems: Models and Spectral Efficiency Performance," IEEE Wireless Commun., vol. 25 , no. 2, pp. 24-31, Apr. 2018.

[23] M. S. Ali, E. Hossain, A. Al-Dweik, and D. I. Kim, "Downlink Power Allocation for CoMP-NOMA in Multi-Cell Networks," IEEE Trans. on Commun., vol. 66, no. 9, pp. 3982-3998, Sept. 2018.

[24] J. Choi, "Non-Orthogonal Multiple Access in Downlink Coordinated Two-Point Systems," IEEE Commun. Lett., vol. 18, no. 2, pp. 313-316, Feb. 2014

[25] J. Farah, A. Kilzi, C. Abdel Nour, and C. Douillard, "Power Minimization in Distributed Antenna Systems Using Non-Orthogonal Multiple Access and Mutual Successive Interference Cancellation," IEEE Trans. on Veh Technol., vol. 67, no. 12, pp. 11 873-11885, Dec. 2018

[26] A. Kilzi, J. Farah, C. A. Nour, and C. Douillard, "New Power Minimization Techniques in Hybrid Distributed Antenna Systems with Orthogonal and Non Orthogonal Multiple Access," IEEE Trans. on Green Commun. and Netw., pp. 1-1, 2019.

[27] J. Ren, Z. Wang, M. Xu, F. Fang, and Z. Ding, "Unsupervised user clustering in non-orthogonal multiple access," in ICASSP 2019 - 2019 IEEE International Conference on Acoustics, Speech and Signal Processing (ICASSP), May 2019, pp. 3332-3336.

[28] J. Zhu, J. Wang, Y. Huang, S. He, X. You, and L. Yang, "On Optimal Power Allocation for Downlink Non-Orthogonal Multiple Access Systems," IEEE J. on Sel. Areas in Commun., vol. 35, no. 12, pp. 2744 2757, Dec 2017.

[29] R. Jain, D. Chiu, and W. Hawe, "A Quantitative Measure of Fairness and Discrimination for Resource Allocation In Shared Computer Systems," DEC Technical Report 301, Sept. 1984.

[30] Y. Saito, A. Benjebbour, Y. Kishiyama, and T. Nakamura, "System-Level Performance Evaluation of Downlink Non-Orthogonal Multiple Access (NOMA)," in 2013 IEEE 24th Annu. Int. Symp. on Pers., Indoor, and Mobile Radio Commun. (PIMRC), Sept. 2013, pp. 611-615.

[31] A. Ghosh, N. Mangalvedhe, R. Ratasuk, B. Mondal, M. Cudak, E. Visotsky, T. A. Thomas, J. G. Andrews, P. Xia, H. S. Jo, H. S. Dhillon, and T. D. Novlan, "Heterogeneous cellular networks: From theory to practice," IEEE Commun. Mag., vol. 50, no. 6, pp. 54-64, June 2012.

[32] H. Tabassum, E. Hossain, and J. Hossain, "Modeling and analysis of uplink non-orthogonal multiple access in large-scale cellular networks using poisson cluster processes," IEEE Trans. on Commun., vol. 65 , no. 8, pp. 3555-3570, Aug. 2017.

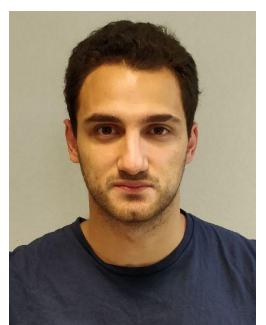

Antoine Kilzi Antoine Kilzi received his computer and communications engineering degree in 2017 from the Lebanese University. He is currently working towards the Ph.D. degree in information and communication engineering at IMT Atlantique. His current research interests include resource allocation, nonorthogonal multiple access and coordinated multipoint systems. 


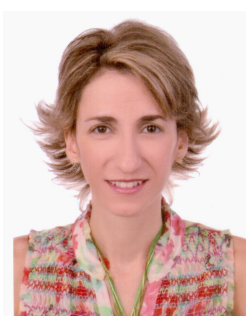

Joumana Farah Joumana Farah received the B.E. degree in Electrical Engineering from the Lebanese University, in 1998, the M.E. degree in Signal, Image, and Speech processing, in 1999, and the Ph.D. degree in mobile communication systems, in 2002, from the University of Grenoble, France. In 2010, she received the Habilitation to Direct Research (HDR) from University Pierre and Marie Curie (Paris VI), France. She is currently a full-time professor with the Faculty of Engineering, Lebanese University, Lebanon. She has supervised a large number of Master and $\mathrm{PhD}$ theses. She has received several research grants from the Lebanese National Council for Scientific Research, the Franco-Lebanese CEDRE program, and the Lebanese University. She has nine registered patents and a software and has coauthored a research book and a hundred of papers in international journals and conferences. Her current research interests include resource allocation techniques, channel coding, channel estimation, interference management, and distributed video coding. She was the General Chair of the 19th International Conference on Telecommunications (ICT 2012), and serves as a TPC member and a reviewer for several journals and conferences.

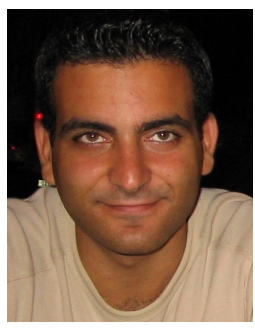

Charbel Abdel Nour Charbel Abdel Nour obtained his computer and communications engineering degree in 2002 from the Lebanese University, his Masters degree in digital communications from the University of Valenciennes, France, in 2003 and his $\mathrm{PhD}$ in digital communications from Telecom Bretagne, France in 2008. From June 2007 till October 2011, he worked as a post-doctoral fellow at the Electronics Department of Telecom Bretagne. He was involved in several research projects related to broadcasting and satellite communications. Additionally during the same period, he was active in the Digital Video Broadcasting DVB consortium where he had important contributions. Starting November 2011, Charbel holds an associate professor position at the Electronics Department of Telecom Bretagne. His interests concern the radio mobile communications systems, broadcasting systems, coded modulations, error correcting codes, resource and power allocation for NOMA, waveform design, MIMO and iterative receivers. Lately, he presented several contributions to the H2020 METIS and FANTASTIC5G projects and to the 3GPP consortium related to coding solutions for $5 \mathrm{G}$.

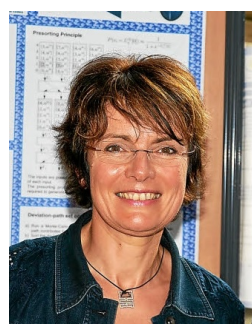

Catherine Douillard Catherine Douillard received the engineering degree in telecommunications from the Ecole Nationale Suprieure des Tlcommunications de Bretagne, France, in 1988, the Ph.D. degree in electrical engineering from the University of Western Brittany, Brest, France, in 1992, and the accreditation to supervise research from the University of Southern Brittany, Lorient, France, in 2004. She is currently a full Professor in the Electronics Department of Telecom Bretagne where she is in charge of the Algorithm-Silicon Interaction research team. Her main research interests are turbo codes and iterative decoding, iterative detection, the efficient combination of high spectral efficiency modulation and turbo coding schemes, diversity techniques and turbo processing for multicarrier, multi-antenna and multiple access transmission systems. In 2009, she received the SEE/IEEE Glavieux Award for her contribution to standards and related industrial impact. She was active in the DVB (Digital Video Broadcasting) Technical Modules for the definition of DVB-T2, DVB-NGH as chairperson of the "Coding, Constellations and Interleaving" task force and DVB-RCS NG standards. Since 2015, she has had several contributions in the FANTASTIC-5G and EPIC H2020 European projects intended for the definition of new techniques for $5 \mathrm{G}$ and beyond. 